\title{
Soft Human-Machine Interfaces: Design, Sensing and Stimulation
}

Wentao Dong ${ }^{1}$, Youhua Wang ${ }^{2,3}$, Ying Zhou ${ }^{2,3}$, Yunzhao Bai ${ }^{2,3}$, Zhaojie $\mathrm{Ju}^{4}$, Jiajie Guo ${ }^{2}$, Guoying $\mathrm{Gu}^{5}$, Kun Bai $^{2}$, Gaoxiang Ouyang ${ }^{6}$, Shiming Chen ${ }^{1}$, Qin Zhang ${ }^{2}, *$, YongAn Huang ${ }^{2,3, *}$

1. School of Electrical and Automation Engineering, East China Jiaotong University, Nanchang, 330013, China

2. State Key Laboratory of Digital Manufacturing Equipment and Technology, Huazhong University of Science and Technology, Wuhan 430074, China

3. Flexible Electronics Research Center, Huazhong University of Science and Technology, Wuhan 430074, China

4. Intelligent Systems and Biomedical Robotics Group, School of Computing, University of Portsmouth, Portsmouth, U.K.

5. State Key Laboratory of Mechanical System and Vibration, Shanghai Jiao Tong University, Shanghai 200240, China

6. State Key Laboratory of Cognitive Neuroscience and Learning, School of Brain and Cognitive Sciences, Beijing Normal University, Beijing, 100875, China

\begin{abstract}
Human-machine interfaces (HMIs) are widely studied to understand the human biomechanics and/or physiology and the interaction between humans and machines/robots. The conventional rigid or invasive HMIs that record/send information from/to human bodies have significant disadvantages in practice for longterm, portable, and comfortable usages. To better adapt to natural soft skins, soft HMIs have been designed to deform into arbitrary shapes, and their bendable, stretchable, compressible and twistable properties offer a huge potential in future personalized applications. This paper presents a survey on various soft HMIs in terms of design, sensing, stimulation as well as their applications. Specifically, tactile / motion / bio-potential sensors are categorized for recording various data from human bodies, while stimulators are discussed for information feedback and motion activation to human bodies. It is anticipated that soft HMIs will promote the interaction among humans, machines/robots and environment to achieve desired coexisting-cooperativecognitive function in a robot system, named as Tri-Co Robot, for the human-centered applications, such as rehabilitation, medical monitoring and human-robot cooperation.
\end{abstract}

Keywords: Human-machine interfaces; flexible/stretchable electronics; tactile sensors; biological healthy monitoring; stimulation feedback

\section{Introduction}

Human-machine interfaces (HMIs) refer to the studies of the two-way transmission of information between humans and machines (such as computers or robots) [1]. Recording and interpreting physical and/or

\footnotetext{
*Corresponding author: Y.A. Huang, yahuang@hust.edu.cn; Q.Zhang, qin.zhang@hust.edu.cn
} 
physiological information of humans is a key point to allow the robots interacting with humans. Conventional rigid HMIs have been widely applied in robotic systems, such as rehabilitation robots [2,3], motion gesture monitoring [4], and biological health monitoring [5]. However, they generally lead to the uncomfortablity of the human body and unstable signal in motion [6-8] by directly laminating onto the skin surface $[9,10]$. Alternatively, soft HMIs based on flexible/stretchable electronics can fulfil the requirements of the nextgeneration HMIs that offer the sensing functions of conventional, rigid technologies but also with the ability to be stretched, compressed, twisted, bent, and deformed into arbitrary shapes [11, 12]. They overcome the fundamental mis-match in mechanical and material properties with human bodies, and thus would realize innovative applications that are impossible for rigid HMIs. It is very important to design the soft HMIs to adapt to the musculoskeletal deformations including local deformation of skin/muscule and global motion of human $[13,14]$. It is beneficial to achieve the expected coexisting-cooperative-cognitive robot (Tri-Co Robot) system for the purpose of rehabilitation, medical monitoring and human-robot cooperation [15].

As illustrated in Fig.1, the soft HMIs for humans interacting with machines/robots or monitoring human health condition can be divided into several categories: i) soft tactile sensors in E-skin for measuring the pressure and temperature of humans and robots; ii) motion sensors for measuring the joint angles and velocities of natural/artificial limbs; iii) electro-physiology sensors, such as EEG and EMG, for trajectory controlling or health monitoring, as the green and blue arrows; and iv) feedback stimulators for applying electrical stimulations to human bodies, as the red arrows.

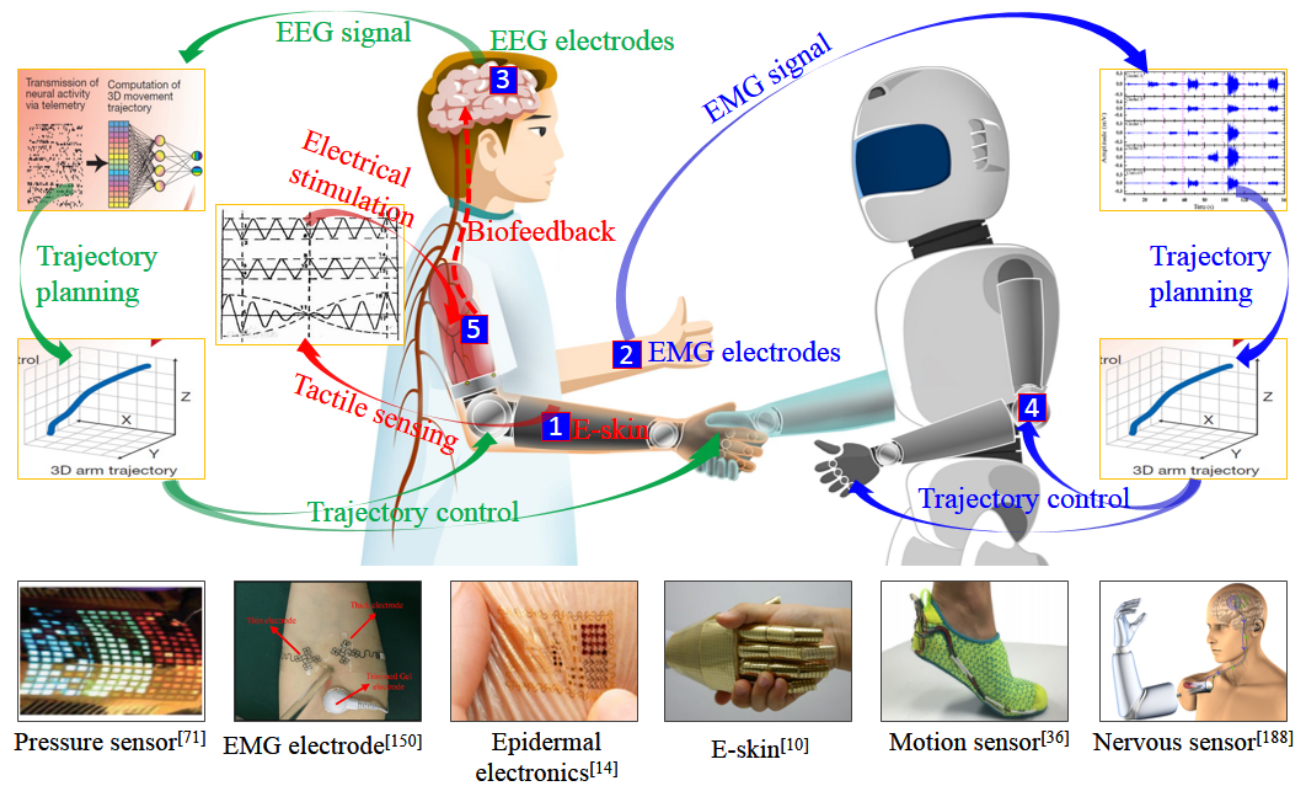

Fig.1 Schematic diagram for humans interacting with the machines/robots, and various flexible/stretchable devices for soft HMIs. Bottom images: "Pressure sensor ${ }^{71,}$, "E-skin ${ }^{10,}$ and "nervous sensor ${ }^{188 "}$ reproduced with permission. Copyright Macmillan Publishers Ltd; "EMG electrode ${ }^{150}$." image

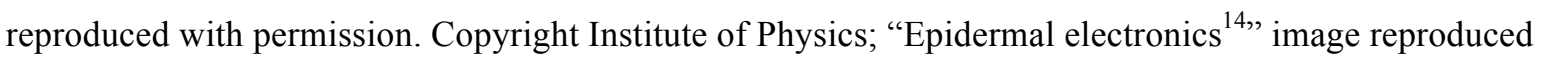
with permission. Copyright American Association for the Advancement of Science; "Motion sensor ${ }^{36 "}$ 
image reproduced with permission. Copyright IEEE.

The soft tactile sensors are designed as superskins with higher sensitivity and time-space resolution to monitor pressure, strain, sound and light information for HMI applications. Soft pressure sensors based on capacitive sensing arrays [13, 16, 17], piezoelectric materials [4], porous pressure sensitive rubber [18], and ionic gels [19] are designed to monitor biological signals, which have been widely applied to pressure visualization and reproducing the tactile sensing abilities. Stretchable strain sensors based on piezoresistive materials [20], $\mathrm{ZnO}$ nanowires [21], metal coils [22, 23], and liquid metals [24] are developed to detect human gestures. Artificial electronic eyes have been extensively applied to robot navigation to assist people for complicated tasks [25-27]. Sound signals acquired by piezoelectric materials [28, 29] and EMG electrodes [30] have also been widely adopted in speech analysis and recognition, which show novel interaction ways for playing computer games.

Motion sensing, including measuring joint angles, linear displacements, velocities and accelerations of humans and robots, is critical for the human-machine interaction to accomplish complicated and dynamic tasks [31]. For example, the artificial limbs integrated with motion sensors can be precisely manipulated and controlled in a closed-loop manner [32]. A large number of soft motion sensors have been designed for HMI applications. Soft joint angle sensors based on graphene, smart gloves, and liquid metal are designed to monitor human gesture and control the robot to reproduce the action, which are widely applied in HMIs [33]. Stretchable strain sensors based on carbon-black [34], liquid metal [35], and ionic gel [19] are developed for skin strain, human motion and gestures detection. Velocity and acceleration sensors are widely applied to measure human gestures and positions, and the corresponding algorithms are proposed for classifications for controlling the external machines via human motion state [36].

Electrophysiology signals from human bodies have also been used in HMI applications, and replaced the conventional electrodes for recordings of electromyography (EMG), electroencephalogram (EEG), and electrooculography (EOG) in monitoring of biological health [37-41]. In the state-of-the-art, the flexible/stretchable bio-potential electrodes are designed and developed to provide long-term and stable biological signal recordings [42-44]. The features extraction and pattern recognition algorithms are proposed to classify the biological signals, and the corresponding control signals are generated through the patterns for controlling the external actuators [45-47].

Additionally, stimulations from electronic devices to humans is another kind of important interaction modes that make humans sense external environment or establish closed-loop control between humans and 
machines/robots via the soft HMIs integrated with stimulators and actuators [48, 49]. Electrical stimulation evokes tactile sensations within the skin at the location of a small, cutaneous electrode by passing a local electric current through the skin to stimulate cutaneous afferent fibers [50,51]. Feedback to humans via different stimulators can be divided into electrotactile stimulation, sound and light stimulation, braille display, and EEG-EMG stimulation. Electrotactile stimulation is realized with the external voltage supplied from electronic devices [20]. Sound and light stimulation are designed for biological feedback to improve humans' speech abilities [52]. Braille display based on electroactive polymer actuators (EAP) is designed and developed for visually impaired people to touch and know the outside world more vividly [53-55]. EEGEMG stimulation is proposed for human rehabilitation with the help of robots. Additionally, there are also researches on rendering sense of taste and smell with electronic devices, such as a taste stimulators and artificial noses [56-58].

Based on different sensing and stimulation techniques, many HMIs have been developed for robots, medical diagnostics, and prosthetic devices. Human-robot interaction based on conventional rigid sensors was firstly studied in 1971 when a prosthetic hand integrated with sensors was designed as a part of body to interact with the surroundings [59]. At the beginning of the 1980s, a HMI system based on an electronic hand with full sensory perceptions was invented to assist surgeries [60]. In 1984, Dario reported the ferroelectric polymer tactile sensors integrated in the prostheses through polyvinylidene fluoride $\left(\mathrm{PVF}_{2}\right)$ transducers [61]. It could mimic the human skin to measure contact pressures and hardness. Johansson designed a sensitive skin which could sense its surroundings, and allow the robot arm to avert potential obstacles and effectively maneuver within its physical environment [62]. In the 1990s, Jiang et al. proposed one flexible sensor sheet for tactile shear force sensing and integrated them on flexible polyimide foils [63]. Tzafestas designed a new piezoresistive tactile sensor system for HMI applications, with equally critical mimicing the mechanical properties of human skin to accommodate its various motions, in addition to the ability of an artificial skin to interact with its surroundings [64]. The soft HMIs increasingly attract much attention recently, including two milestone phases as shown in Fig.2.

(1) From 2000 to 2010. Flexible Microprocessors and microsensors were widely designed to flexible HMIs which accelerated the development of electronic skin (E-skin) significantly ranging from robotics to healthcare. Someya et al. developed flexible organic field-effect transistors (OFETs) for large-area integrated pressure-sensitive sheets with active matrix readout $[65,66]$, and the stretchable active-matrix organic light emitted diodes (AMOLED) for large-area integrated pressure-sensitive sheets pressure visualization with 
pressure-sensitive rubber $[67,68]$. A cybernetic hand was integrated with infrared radiation sensors to control hand motions. Rogers et al. designed flexible artificial electronic eyes which provided an effective way for robots to connect and communicate with people [69]. Bao et al. investigated highly sensitive capacitive pressure sensors with microstructural elastomeric dielectrics for large sensitive mechanical force sensing [16]. The flexible optoelectronics including light-emitting diodes (LEDs) and organic photovoltaics (OPVs) were integrated with human skin to show the skin pressure distribution [70].

(2) From 2010 to the present. Stretchable HMI has been attracted more and more attentions. The motion features were detected by the soft electronics integrated with multi-strain sensors to control the external actuator [20]. Javey et al. designed an interactive HMI system integrated with pressure sensitive skin, organic transistor array and LEDs [71], and the pressure distribution was provided to interact with external surroundings [72]. A wearable interactive HMI based on pressure and strain sensors was applied to personal mobile electronics and the Internet of Things [73]. Stretchable EMG electrodes were designed with serpentine structure to recognize different human gestures, and different control commands were generated to the external actuators [74]. The artificial skin integrated with the EMG sensor, strain sensor, and pressure sensor, used as prosthetic skin, was designed to operate complicated tasks, such as grasping the cup, and tapping on the keyboard [75]. The acoustic sensor laminated onto the human throat collected the features signal with different speech and generated control commands to control the computer game [76]. Transparent $\mathrm{ZnO}$ sensors were designed and fabricated to recognize the gestures of different fingers without power supply [77]. A self-powered HMI with cut-paper-based self-charging power unit was used to practical and medical applications by Wang et al. [12, 78, 79]. Transparent HMI with porous pressure sensitive rubber sensors and strain gauges were designed to control a robot arm remotely [18], and the epidermal electronic system with the EMG sensor, strain sensor, humidity sensor, and temperature sensors was also used as an HMI to control the robot arm [80]. 


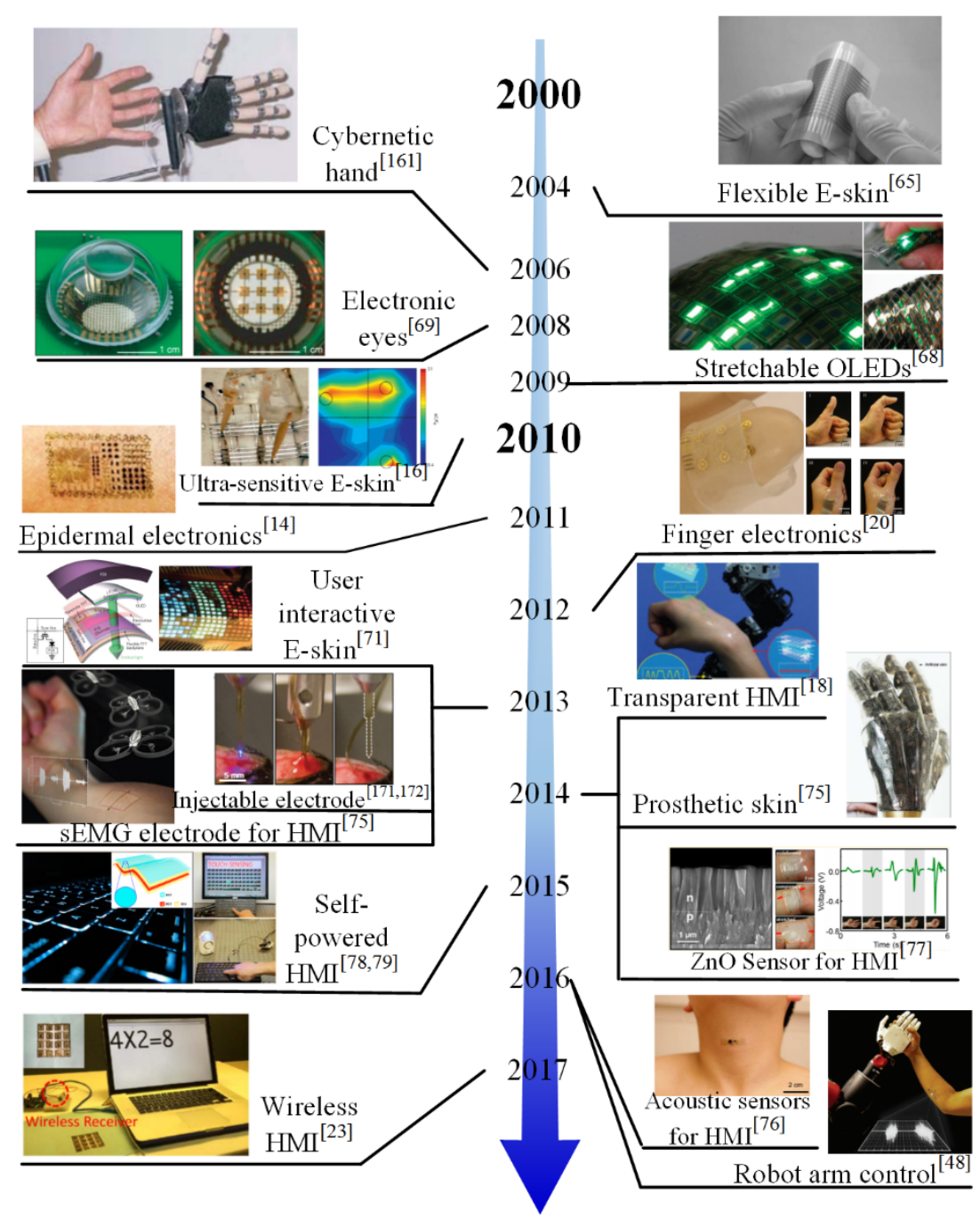

Fig.2 Evolution of HMI based on flexible/stretchable electronics. "Tactile sensor system for robot control $^{66 "}$ " image reproduced with permission. Copyright IEEE. "Flexible E-skin ${ }^{65}$ " image reproduced with permission. Copyright, American Association for the Advancement of Science. "Electronic eyes" "Stretchable OLEDs ${ }^{67 ", ~ " U l t r a-s e n s i t i v e ~ E-s k i n ~}{ }^{16,}$, "User interactive E-skin ${ }^{71 "}$ Prosthetic skin ${ }^{75, ", ~ a n d ~}$

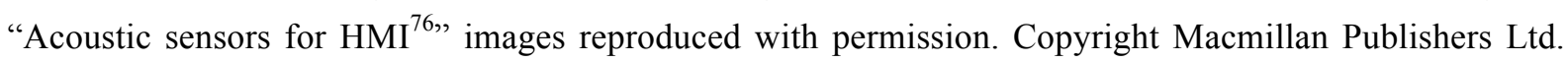
"Injectable electrode ${ }^{178-179, " ~ i m a g e ~ r e p r o d u c e d ~ w i t h ~ p e r m i s s i o n . ~ C o p y r i g h t ~ A m e r i c a n ~ A s s o c i a t i o n ~ f o r ~ t h e ~}$ Advancement of Science. "Finger electronics ${ }^{20 "}$ image reproduced with permission. Copyright Institute of

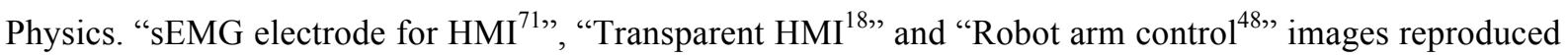

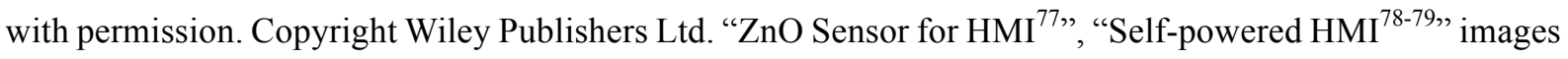

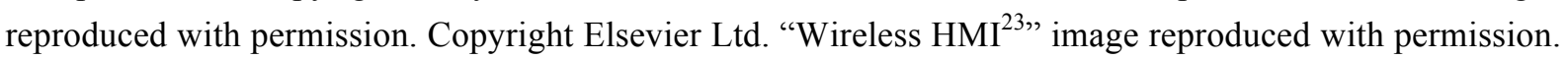
Copyright American Chemical Society.

There were several review articles about the flexible/stretchable electronics which have been applied to soft robot, biological healthy monitoring, electronic skin (E-skin), and detection of human hand motion [3, 81-87]. Lu discussed flexible and stretchable electronics for soft robot [88]. Bao et al. introduced the brief history and development of E-skin, and coexistent problems about its designs and applications [10]. Wang et al. reviewed the recent process of E-skin with multi-mode force sensing, temperature, and humidity detection, as well as self-healing abilities [89]. However, most of these surveys mainly focused on the designs and 
developments of the stretchable devices in different applications, soft HMIs are not systemically reviewed for the design and applications for human-machine interaction. The stretchable electronics technology enables the next generation of electrodes for soft HMIs [78]. This paper reviews the development of soft HMIs based on stretchable electronics, including design methods, sensing/stimulation principles, and interaction applications. Material and structural design for stretchability are introduced in Section 2. Three typical HMI modes and their applications are reviewed in Section 3. Stimulation from electronic devices to humans is introduced in Section 4. The whole paper is concluded with remarks in Section 5.

\section{Structural design of flexible/stretchable components}

Material selection and structural design are important to improve the stretchability of HMIs. Recent progresses on the developments of stretchable materials have enabled a number of intrinsically stretchable devices [90-93], such as liquid metal, hydrogels and rubber. Epidermal strain sensor based on liquid metal and PEIE-polydimethylsiloxane (S3-PDMS) elastomer substrate could be stretched to 50\% and shows excellent compatibility with human skin [24, 94]. PDMS microfluidic devices were also adopted in biomedical applications $[35,95]$, where PDMS was widely used in the soft devices, due to the advantages of softness, stretchability, transparency, easy fabrication, bio-compatibility, chemical inertness, stability, and adhesive [96]. Organic polymer with mechanical and electrical self-healing properties was applied to electronic skin in soft robotics and biomimetic prostheses [97].

On the other side, structural design is critical for stretchable devices based on inorganic material with extreme strain of $1-2 \%$. It has experienced three symbolic stages: stretchablity, conformability and stability. (1) Geometry design strategies is adopted to improving the stretchability without reducing the electrical performance of the electronic devices. (2) When the soft electronics laminated onto the skin surface or soft tissue surface, conformability should also taken into consideration for more accurate bio-potential signal recordings. (3) The in-plane deformation interconnect would be more stable during the stretched process.

(1) Several approaches were utilized to fabricate stretchable interconnect structures (Fig.3(a)): patterned thin film on the prestrained substrate to generate nonplanar buckled structures (left frame) [98, 99]; and serpentine film on stretchable substrate, such as wrinkled, serpentine structures (center frame) [100-102], and self-similar serpentine structure (right frame) [12, 101, 103-106]. The serpentine interconnects were usually designed to be freestanding for large stretchability [107]. However, the freestanding format was a challenge for encapsulation. Soft microfluidic assembly technique is studied to address this challenge [108]. The self- 
similar serpentine structure was enhanced version of serpentine design for hyper-stretchable devices, simutaneously with high areal coverage shown in Fig.3(f) [12, 101, 103-106, 109]. Structural optimized strategies were proposed to improve the stretchability of the rectangle electrode $[74,110]$. Topology optimization strategies were proposed to optimize the stretchability of electronic devices the with soft mechanism designs $[111,112]$.

(2) Beyond the stretchability, the conformability should be considered into the design strategies of the soft HMIs. The soft HMIs laminated onto the skin surface should follow the motion of the soft skin surface for more accurate bio-potential signal recordings. Fig.3(b) shows that the soft devices contact with the skin surface conformally for reducing motion artifacts $[74,113]$. Stretchable electronics with low bending stiffness and strong adhesion are able to promote conformal contact with human skin, yet provide conformal contact to the soft skin surface, and the critical design strategy is proposed to keep conformal contact between the skin and soft electronics [114, 115]. In particular, conformability is key to high-performance functioning electronics for HMI application.

(3) The stability was another important factor for the design of soft HMIs during being stretched. There were two buckling modes: out-of-surface buckling and in-surface buckling [116]. The former affects the electrical performance as the stretchable interconnects would be detached from the substrate. The in-surface deformation interconnect was designed for stretchable electronics with thick bar geometries to yield scissorlike deformations modes as shown in Fig.3(c) $[99,104]$. It was still in surface during the stretching process with thick bar design methods. 
(a)

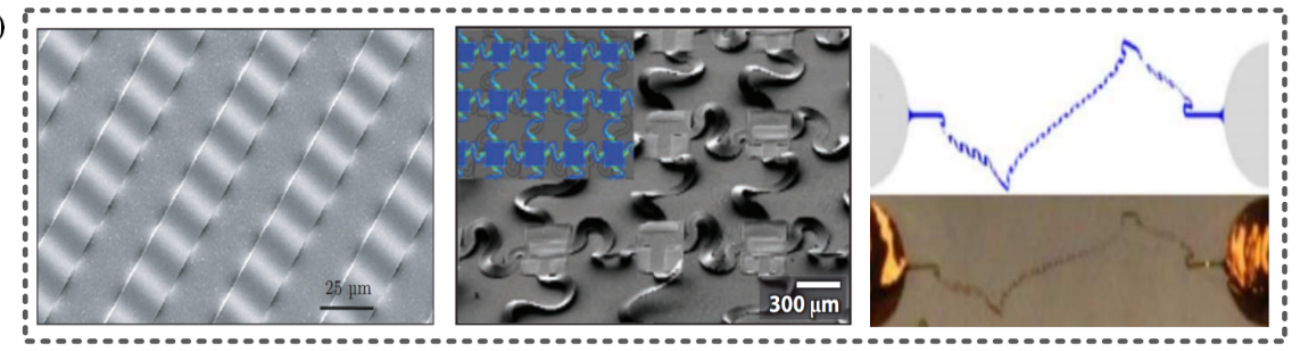

(b)

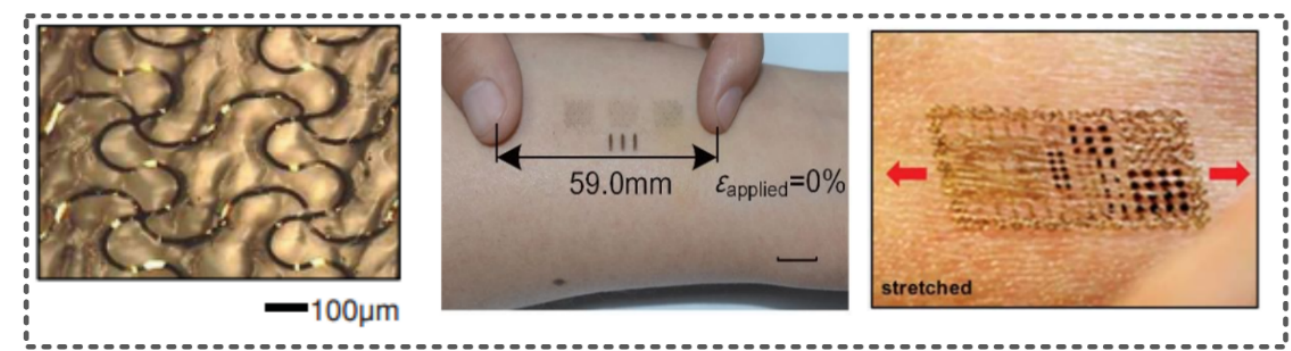

(c)

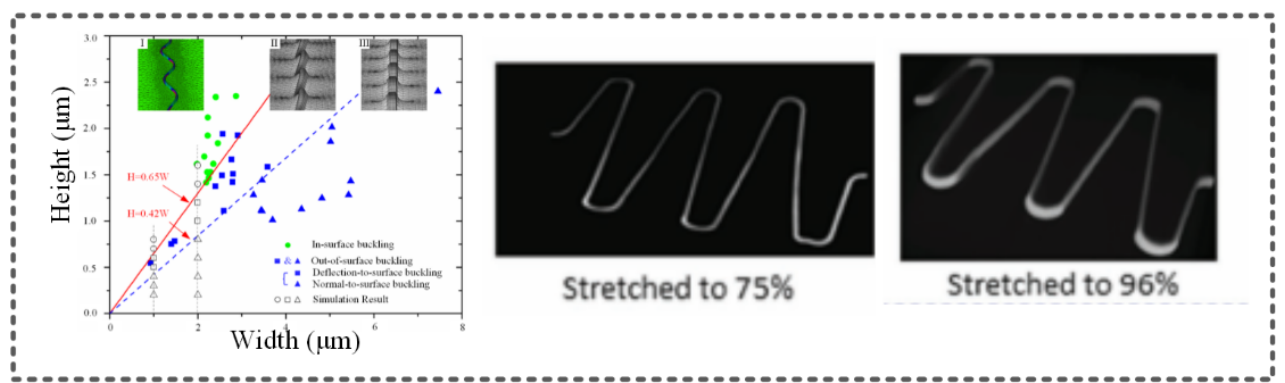

Fig.3 (a) Stretchability design for HMIs [100, 101, 117]; Adapted with permission. Copyright Macmillan Publishers Ltd; (b) Conformability [118], Adapted with permission. Copyright Institute of

Physics; (c) Stability design for stretchable electronics during been stretched [116], Adapted with permission. Copyright Wiley Publishers.

\section{Sensing from humans via HMIs}

\subsection{Tactile sensors}

Various soft tactile sensors have been designed and categorized by the functions such as pressure sensors, strain sensors and acoustic sensors. Pressure is one of the key physical parameter to evaluate the human sensing ability. Several kinds of pressure sensors have been designed for HMI applications, such as capacitive sensors [16], pressure sensitive rubber (PSR) sensors [119], piezoelectric pressure sensors, liquid metal sensors, and ionic gel sensors.

Capacitive sensors were designed to detect the force changes with variable effective area or distance between the two conductive plates via the external pressure [120]. Bao et al. designed a transparent and stretchable capacitive sensor array based on carbon nanotubes on elastic substrate that was sensitive to both pressure and strain along the directions in length and thickness (Fig.4(a)) [16]. The sensitivity of the capacitive sensor array depends on the pyramid-structured PDMS which increases the air voids between the 
PDMS and the organic semiconductor layer. It also provided an effective way of pressure visualization with high sensitivity. Fig.4(b) depicted a flexible and highly functional capacitive pressure sensor for measuring different kinds of forces in different directions [121]. It provides an effective method to measure pressure, shear and torsion.

(a)

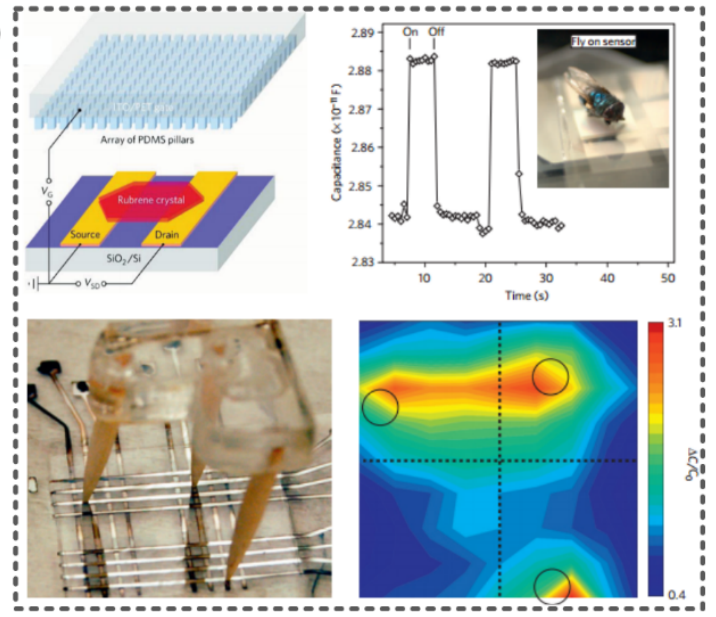

(b)

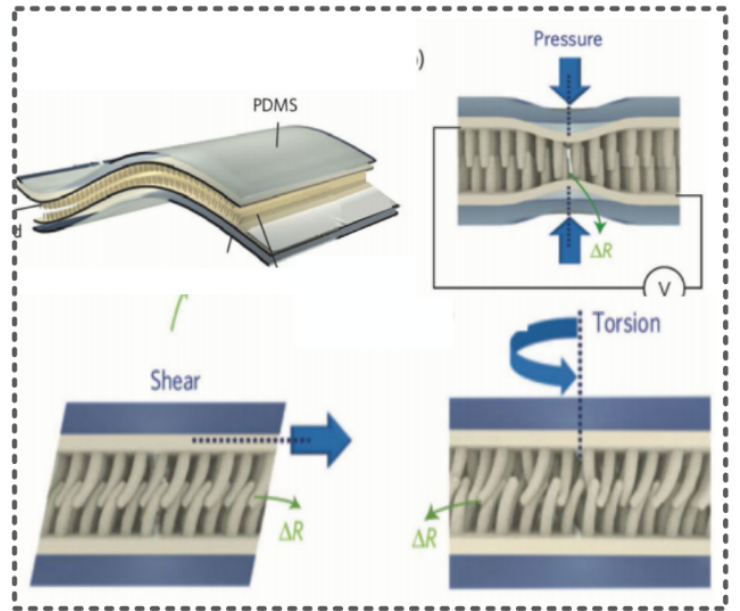

Fig.4 Soft HMIs based on capacitive pressure sensitive sensors. (a) Ultra-sensitive pressure sensor with PDMS microstructure [16]. Adapted with permission. Copyright Macmillan Publishers Ltd; (b) Mutlifunctional capacitive pressure sensor [121]. Adapted with permission. Copyright Macmillan Publishers Ltd.

Soft PSR sensors were another important way for the pressure sensing to HMI applications. Javey et al. developed a tactile sensing glove for capturing a variety of comprehensive hand motions, such as holding, gripping, grasping, squeezing and so on (Fig.5(a)) [71, 122]. To realize the visual display of pressure distribution, a user-interactive E-skin based on PSR sensor was also developed for pressure visualization. The OLEDs were laminated between the active matrix transistors and the PSR layer, which could be turned on with a decrease in resistance of the rubber which clearly gives a promising application in the force visualization for any pressure applied to the smart glove. Wearable PSR-based pressure and strain sensors were developed on a commercial elastomeric patch for control the mobile robot motion as shown in Fig.5(b) [119]. The strain gauge was composed of two channels and the pressure sensor consists of four channels for designating directions and control speeds. The commands were remotely delivered by mechanical motions through the six channels. Each signal measured by the pressure sensors or strain gauges would generate different control command for the motion of the robot [123]. It is usually able to become thinner than capacitive sensors. 
(a)

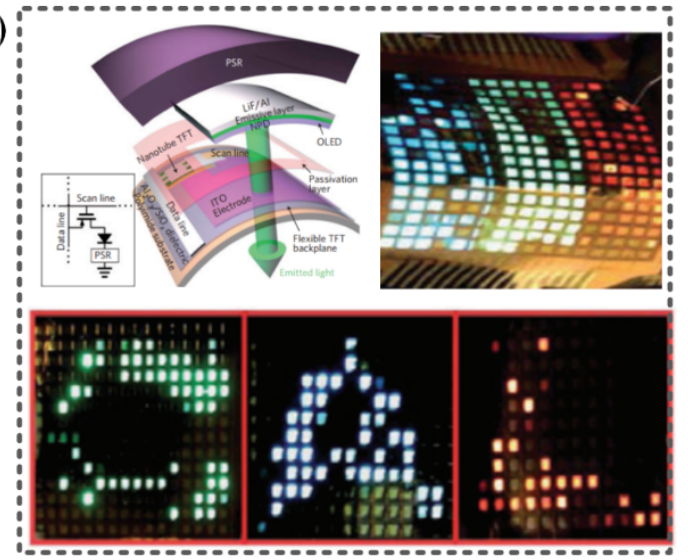

(b)

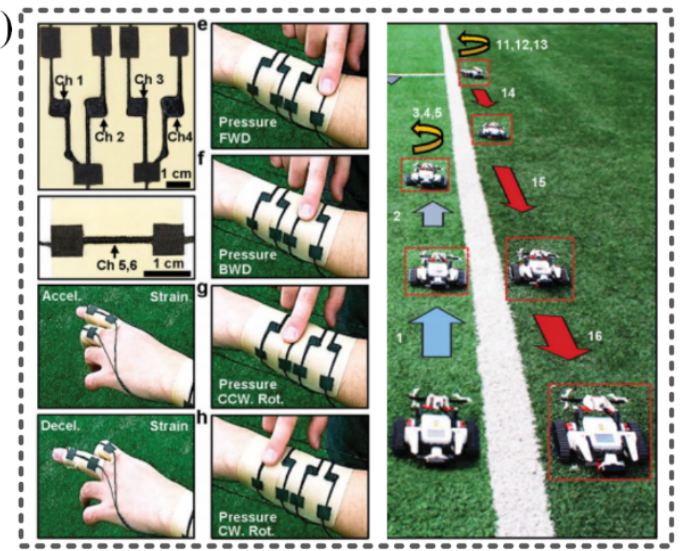

Fig.5 Soft HMI based on wearable PPSR-based pressure sensor.(a) User-interactive E-skin [71] Adapted with permission. Copyright 2013 Macmillan Publishers Ltd; (b) Wearable PSR-based pressure sensor [119], Adapted with permission. Copyright Wiley Publishers Ltd.

Piezoelectric materials have been widely applied to monitor the pressure of the human motion for HMI applications. Fig.6(a) introduced the transparent and self-powered $\mathrm{ZnO}$ nanowire sensor for gesture recognition and HMI applications without power supply to the electronic system [124]. The piezoelectric $\mathrm{ZnO}$ sensors were designed with high sensitivity which could map the pressure distribution [125]. Selfpowered pressure-sensitive triboelectric sensor matrix was designed and developed for real-time tactile mapping, and it has the potential to HMI applications [126] as shown in Fig.6(b). A stretchable triboelectricphotonic smart skin was reported to enable multidimensional tactile and gesture sensing for a robotic hand [127]. Piezoelectric sensors is more sensitive for dynamic motion, and can realize self-powered system which is suitable for wearable and soft HMIs.
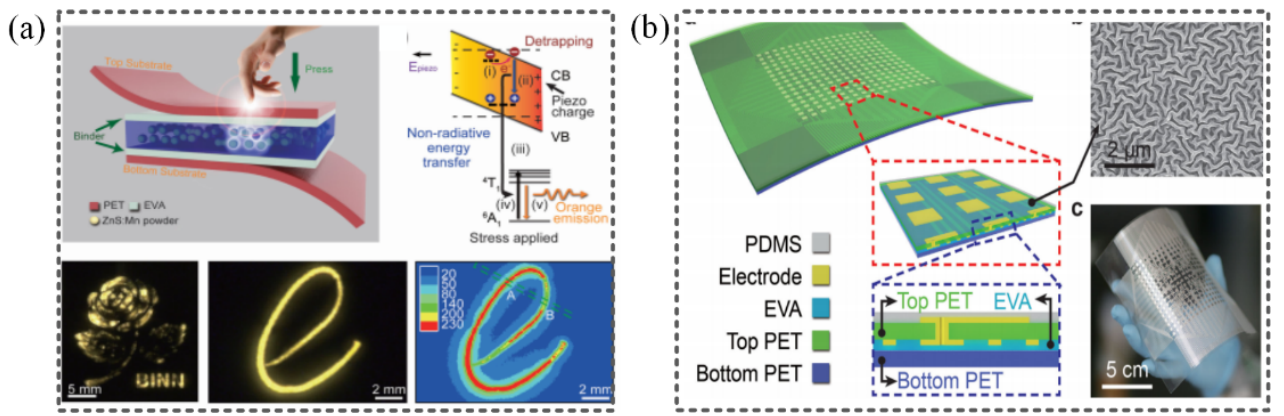

Fig.6 Soft HMI based on wearable piezoelectric pressure sensor. (a) Transparent and self-powered $\mathrm{ZnO}$ nanowire sensor [124], Adapted with permission. Copyright Elsevier Ltd.; (b) Self-powered pressuresensitive triboelectric sensor [126], Adapted with permission. Copyright Macmillan Publishers Ltd.

Conductive ionic hydrogels were also used to pressure monitoring which could satisfy the soft property of the skin surface. Hydrogels were soft like tissue and very stretchable, where ions could be dissolved into water as ionic conductors [128]. A flexible transparent touch panel based on conductive liquid channels was developed to detect the load which is mounted on the round surfaces [129]. Kim et al. constructed a novel 
ionic touch panel with one conductor layer and four electrodes with one at each corner, as shown in Fig.7 [130]. This touch panel could measure continuous plane locations, which is different from the conventional multi-electrode arrays. Utilizing the touch panel, people could write words, play music and chess. The pressure sensory sheet based on ionic conductors ("ionic skin") with highly stretchable, transparent, and biocompatible properties was designed to measure pressure and strain information [19].

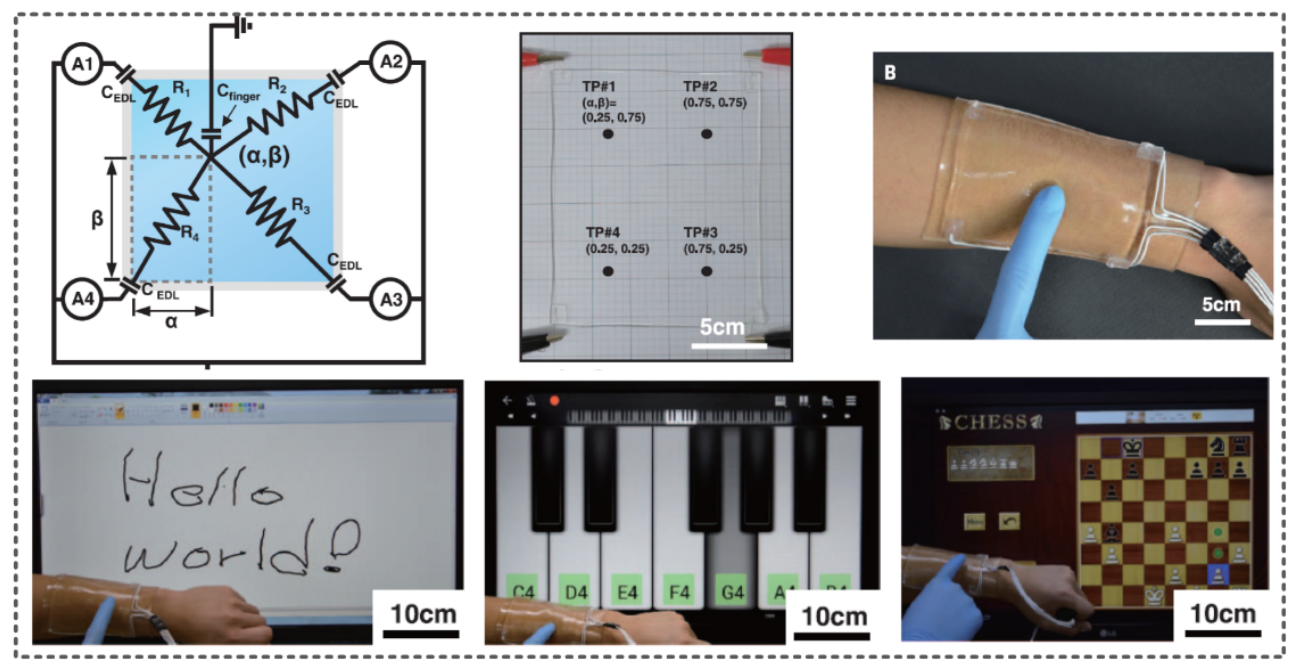

Fig.7 Soft HMI based on transparent ionic gel pressure sensor [130]. Adapted with permission. Copyright American Association for the Advancement of Science.

Strain sensor was designed to monitor the deformation of the human body for human-machine interaction [123, 131]. Strain sensors were utilized to catch multi-finger gestures, as shown in Fig.8(a) [77]. Through the motion of different fingers, various control commands can be generated for external actuators. Fig.8(b) depicted the stretchable Si gauge was designed as a tube to detect the finger motion[20], which contacts with finger conformally. A wearable force sensor array, where the touch signal was detected by conducting two transparent electrodes when the pressure was acquired by Si strain gauges. The liquid metal was encapsulated by elastomer channels for monitoring skin strain, which had excellent electrical and mechanical properties, such as the approximate conductivity with solid metal, and stretchability due to the soft elastomers and liquidity of the liquid metals. 

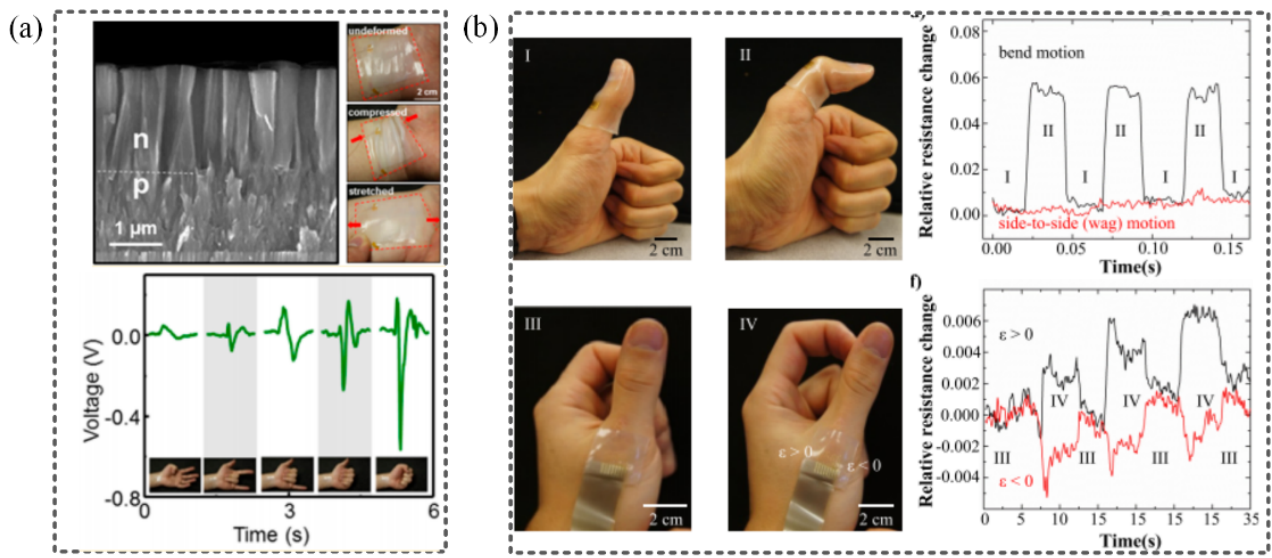

Fig.8 Strain sensor for HMI. (a) Self-powered strain sensor [77], Adapted with permission. Copyright Elsevier Ltd.; (b) Strain sensor based on semiconductor silicon ribbon [20]. Adapted with permission. Copyright Institute of Physics.

Acoustic sensors were integrated into E-skin, to provide a function of sound recording. The epidermal mechano-acoustic devices with multiple sensors were compatible with the soft curvilinear skin, which captures and recognizes the speech via the voice-activated HMI. Fig.9 depicted an epidermal mechanoacoustic sensor which recognized the human voice and controls the motion of the robot via different voices [76]. The devices could simultaneously capture acoustic vibrations from the vocal cords, such as speaking "left," "right," "up," and "down". This feature could allow the epidermal acoustic sensor to be used for communication in noisy environments. Acoustic sensors based on piezoelectric nanofibres with high sensitivity was fabricated by electrospinning for recognizing human voices[132]. Acoustic sensors provide an innovative method for wearable sound recorders and voice interaction with robots/machines.

(a)

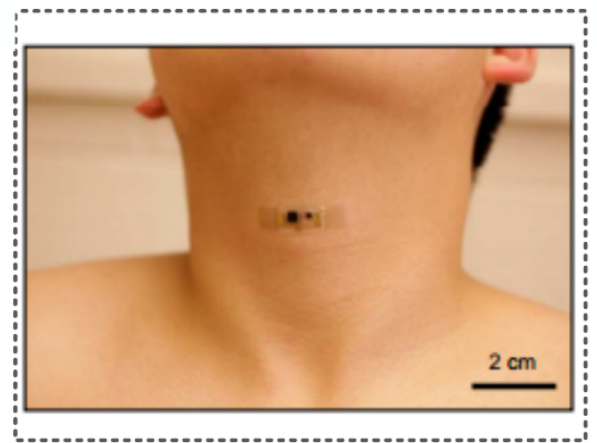

(c)

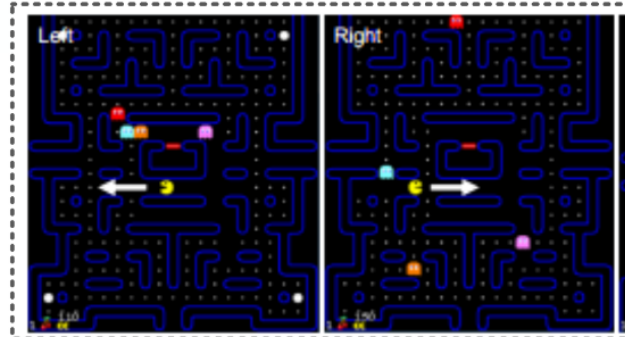

(b)
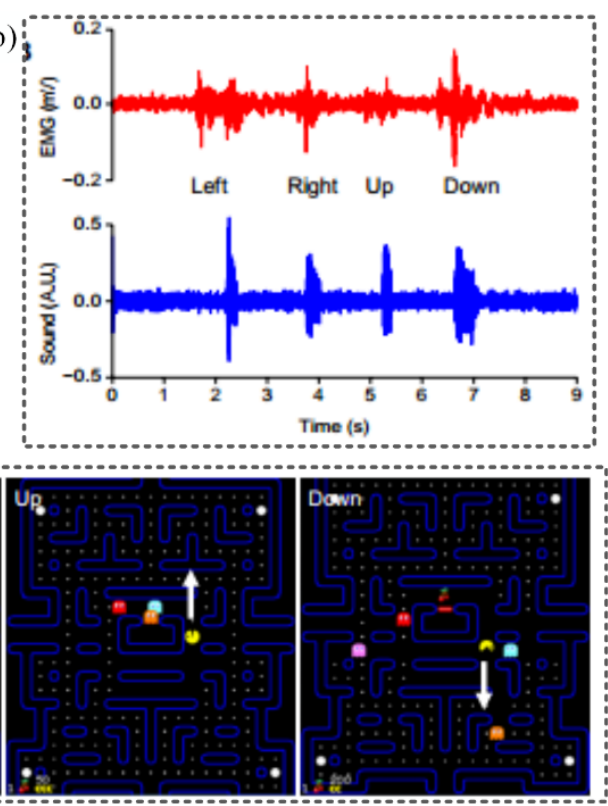

Fig.9 Mechano-acoustic sensor for HMI application [76]. Adapted with permission. Copyright American Association for the Advancement of Science. (a) Epidermal mechano-acoustic sensor laminated 
onto the throat; (b) Classification signals from different speech via the acoustic sensor; (c) Control the computer game.

\subsection{Motion sensors}

Coordination and collaboration between humans and robots require motion sensing for complicated tasks. Soft sensors producing signals conforming to limb/joint rotations or soft-tissue deformations can be used to interpret human body motions from aspects of kinematics (angle, velocity and acceleration) [133], kinetics (pressures and forces) [134] and energy/power (muscle forces and deformations), which can be employed for motion intent recognition and robot control $[135,136]$.

A wearable sensing system based on capacitors embedded in soft cuffs was designed to monitor the shape changes of leg muscles during walking for locomotion mode recognition [135] and gait phase estimation [136] of transtibial amputees. The robust capacitive signals provided a promising alternative for the control of exoskeleton/orthosis. Fig.10(a) depicted a rehabilitation system which enables patients with muscular dystrophy to perform repetitive rehabilitative tasks and regain hand functions. Joint angle sensors were embedded into the rehabilitative system to offer better patient outcomes through therapies [137]. The patients could regain hand functions with the help of the HMI therapy based on joint angle sensors. A prosthetic hand integrated with artificial skin was highly compliant, and mechanically coupled to the curvilinear surface of the prosthesis shown in Fig.10(b) [75]. The prosthetic hand integrated with motion joint angle sensors could monitor strain and joint angle information during the hand movements. With the help of the sensors, the prosthetic hand can perform like a human hand for grasping, fisting, and writing, which demonstrated a good example in the HMI application.
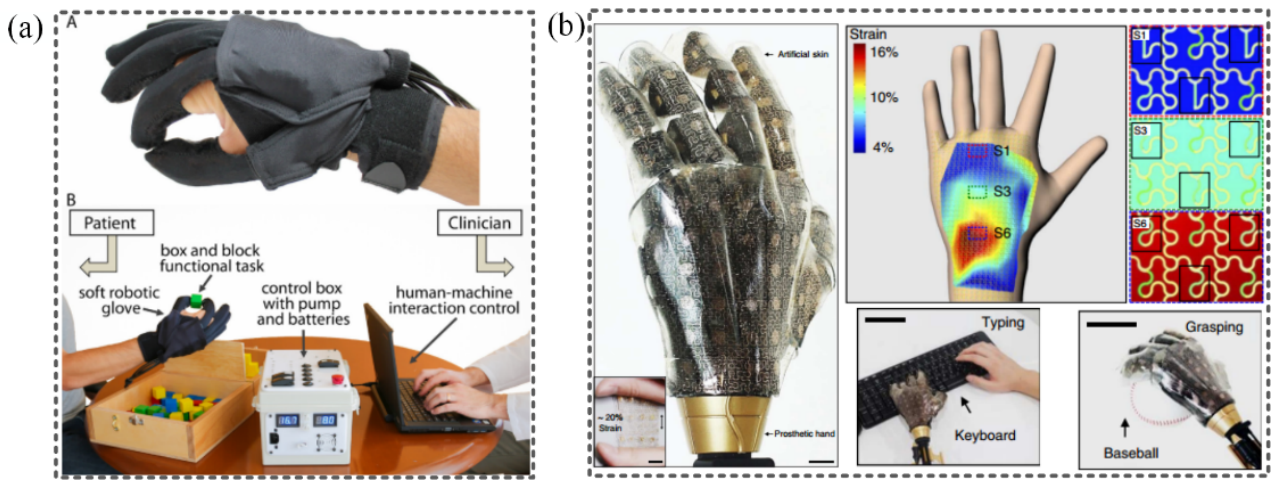

Fig.10 Joint angle sensor for HMI application.(a) Rehabilitative system with joint angle sensors [137];

Adapted with permission. Copyright IEEE. (b) A prosthetic hand with multi-functional sensors [75]. Adapted with permission. Copyright Macmillan Publishers Ltd.

Human motion would induce the skin deformation, and strain sensors are widely designed to detect the human skin deformation. Deformation information from different parts of the body (wrist, fingers, hands and so on) were sensed by stretchable sensors. Fig.11(a) depicted the interactive HMI system with the ultrathin, lightweight, and stretchable nature of devices which were developed to control the motion of the robot arm [138]. The wrist deformation were measured to control the motion of the robot. The finger joint motion angles 
were detected by an epidermal strain sensor with the liquid metal (Fig.11(b)), and several features were extracted with the different bending angles for the potential HMI application [24]. Fig.11(c) depicted a PDMS "smart glove" with multiple embedded microfluidic diaphragm pressure sensors monitoring human joint angles. The smart glove was capable of providing dynamic responses toward a variety of hand motions such as holding, gripping, grasping, squeezing, lifting, moving or touching objects [139]. Additionally, a sensitized garment with 52 channels of signals derived from a user's shoulder and elbow movements was developed to control a virtual robotic wheelchair [140].

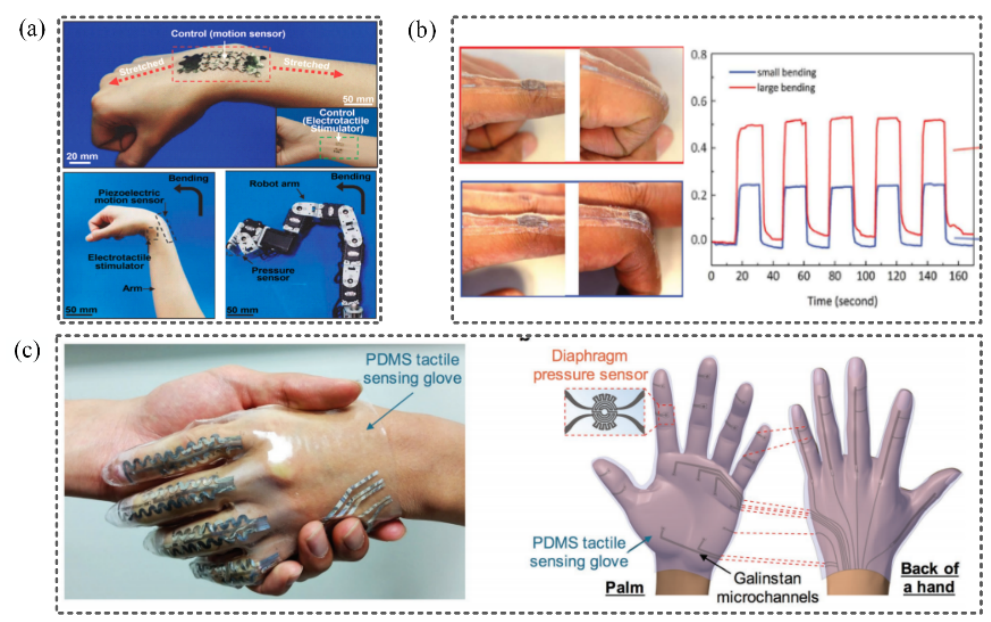

Fig.11 Joint angle sensor for HMI application. (a) Interactive human machine interface for robot arm control [138] Adapted with permission. Copyright Wiley Publishers Ltd.; (b) Epidermal strain sensor with liquid metal for finger motion detection [24]. Adapted with permission. Copyright Wiley Publishers Ltd.; (c) A PDMS "smart glove" with embedded microfluidic pressure sensors [141]. Adapted with permission.

\section{Copyright IEEE.}

Wearable, skin-mountable, and printed strain sensors can be used to detect the body deformation signals at different parts of body in a precise manner. Fig.12(a) depicted the sensors attached to the wrist and elbow to record human deformation signals during the sporting process. Muscle activities were detected by recording muscle bulging in the forearm using a flexible tactile sensor, through which Gaussian process regression was carried out to predict wrist, hand and single-finger activation for controlling prosthesis and assistive robots [142]. Fig.12(b) depicted that thin carbon-black-doped PDMS was designed as the strain gauges featured with its high resistivity and strong dependence on strains, which was used for human gesture detection [34]. Microfluidic stretchable strain sensor based on printed liquid alloys was designed to monitor human gestures which provides large deformation ability [143]. This kind of strain sensors can stick on skin to measure deformation without mechanism. 
(a)

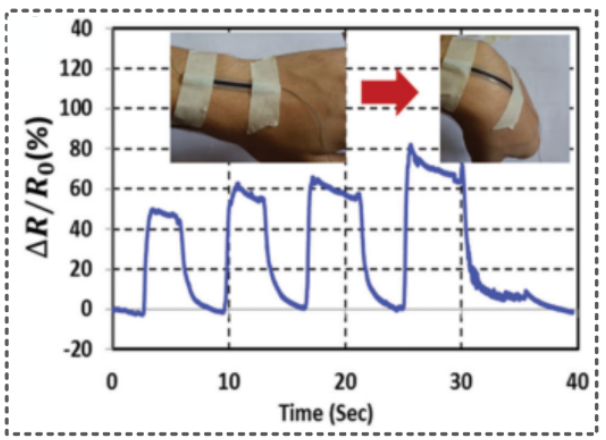

(b)

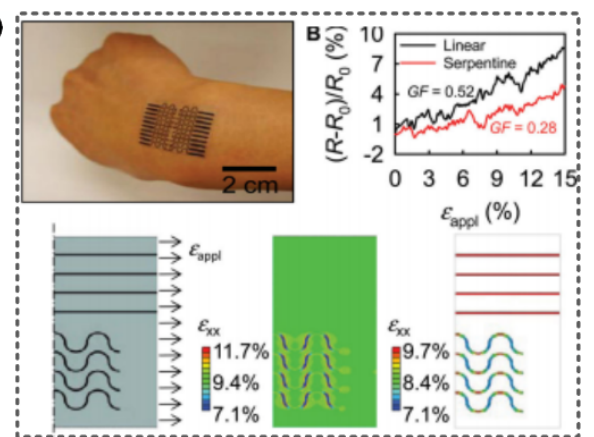

Fig.12 Soft strain sensor for HMI application. (a) Wearable strain sensors [142]. Adapted with permission. Copyright Multidisciplinary Digital Publishing Institute; (b) CB-PDMS based strain gauge [34]. Adapted with permission. Copyright Wiley Publishers Ltd.

The velocity and acceleration sensors are designed to sense the kinetics of human bodies. Fig.13(a) depicted a smart shoe integrated with velocity sensor for detecting human motions. Different characteristic signals were classified with different human motion modes, and different human gestures are generated to interact with surroundings [36]. A printed flexible multiplexed physical sensor was mounted onto the skin surface for human motion signal recordings as shown in Fig.13(b). The flexible sheets integrated with chemical sweat $\mathrm{pH}$ sensor and ion sensitive field-effect transistor were demonstrated in real-time human motion monitoring. A skin-inspired highly stretchable and conformable matrix network (SCMN) was developed multi-sensing functions including but not limited to temperature, in-plane strain, humidity, light, magnetic field, pressure, and proximity. It was incorporated on a personalized intelligent prosthesis to demonstrate its capability in real-time spatial pressure mapping and temperature estimation [144]. A sensing system was developed for real-time monitoring the fastening-strap pressure between human bodies and exoskeletons to reduce risks of skin ulcers, necrotic tissue [145].

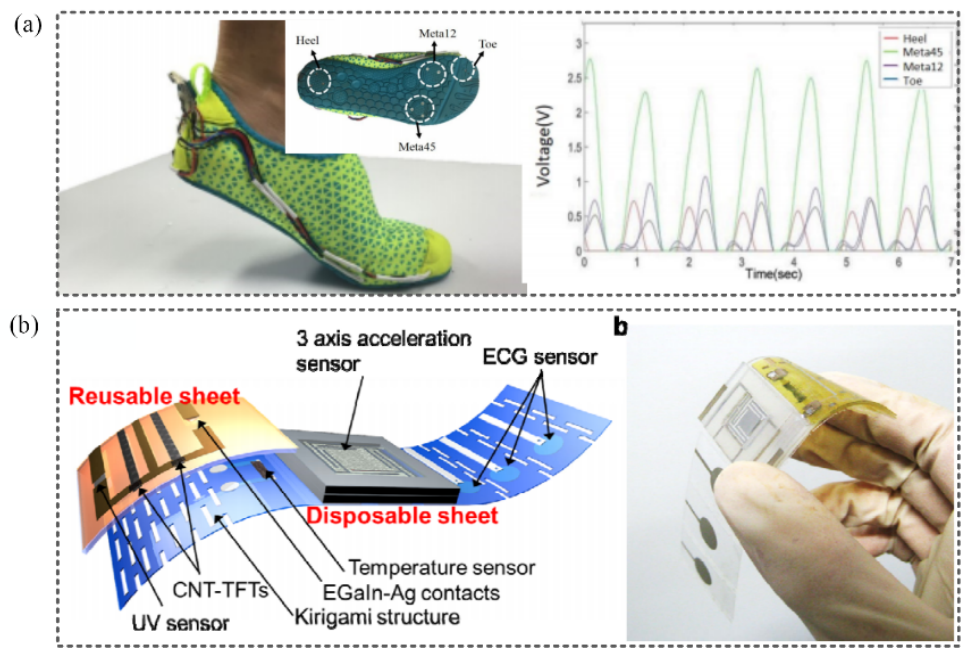

Fig.13 Motion sensor for HMI application. (a) A smart shoe integrated with velocity sensor [36]. Adapted with permission. Copyright IEEE; (b) Printed flexible three-axis acceleration sensor [144]. 


\subsection{Electrophysiology sensors}

Muscle contraction can generate electrical potentials, called as electromyography (EMG) signals, which could be noninvasively acquired by the sEMG electrodes[74, 146-148]. Signals obtained during bimanual gestures such as 'squeeze fists', 'bend wrists to the left', 'bend wrists to the right', and 'bend wrists outward' generated four characteristic sEMG patterns, as shown in Fig.14(a). Linear discriminant analysis (LDA) method was used to classify the features into discrete gestures [149], and it demonstrated that the motion of the four-rotor aircraft is controlled by the EMG sensor. Fig.14(b) depicted a stretchable sEMG patch which could realize the robot hand mimicking human gestures for robot manipulation via eight gestures of a human hand [150]. The sEMG signals were captured from different hand gestures as shown in Fig.16(b). The collected signals from the eight gestures were used for training and classification for robot manipulation.
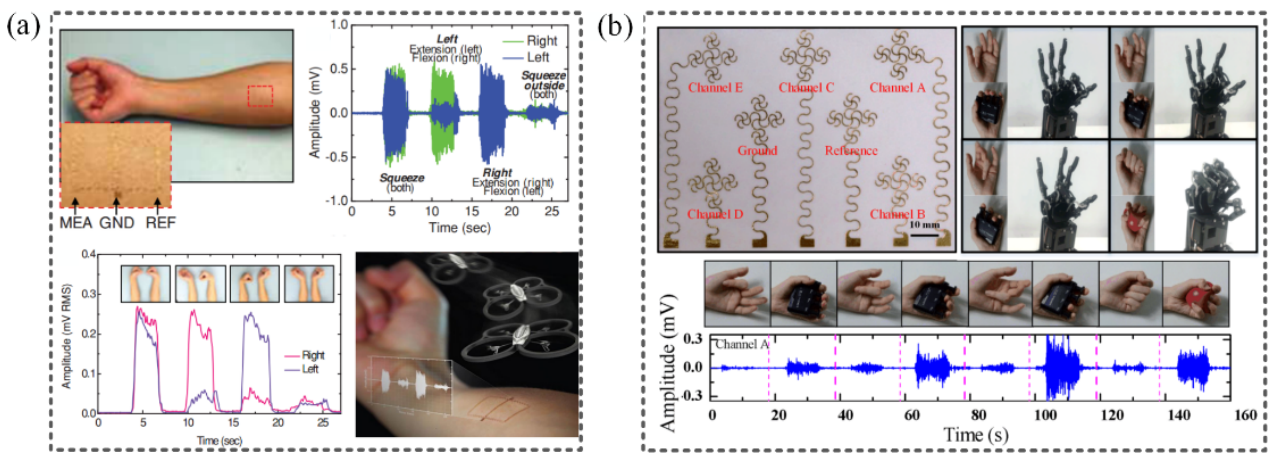

Fig.14 Stretchable EMG electrode for HMI to control the robot motion. (a) Motion control of the fourrotor aircraft via the electrode placed on the forehand for extracting feature signal from different gestures

[149]. Adapted with permission. Copyright Wiley Publishers Ltd; (b) Stretchable multichannel sEMG patches for robot manipulation via eight gestures of the hand. Adapted with permission. Copyright Institute of Physics.

The EOG sensor mounted on a region of the forehead that was prepared by exfoliating the stratum corneum with tape yielding reproducible and high-quality results, as demonstrated in alpha rhythms recorded from awake subjects with their eyes closed [14,151, 152]. The expected feature at $0 \sim 10 \mathrm{~Hz}$ could be seen in the Fourier-transformed data as shown in Fig.15(a) [153]. The signal-to-noise ratios were comparable with those of conventional, rigid bulk electrodes with conductive coupling gels. Fig.15(b) depicted the EOG systems with three modules: the sensor for EOG acquisition, the acquisition instrument, and the host computer control system [154]. The signals were recorded by a commercial amplifier system, including data processing and a graphic user interface. EOG signals of eye movements (blink, upward, and downward) were recorded for obtaining high classification performance by the Fourier transform method. The bioelectronics system with a high-quality recording of EOG signals shows a great potential application in long-term, wearable, and clinically applicable HMIs. 
(a)
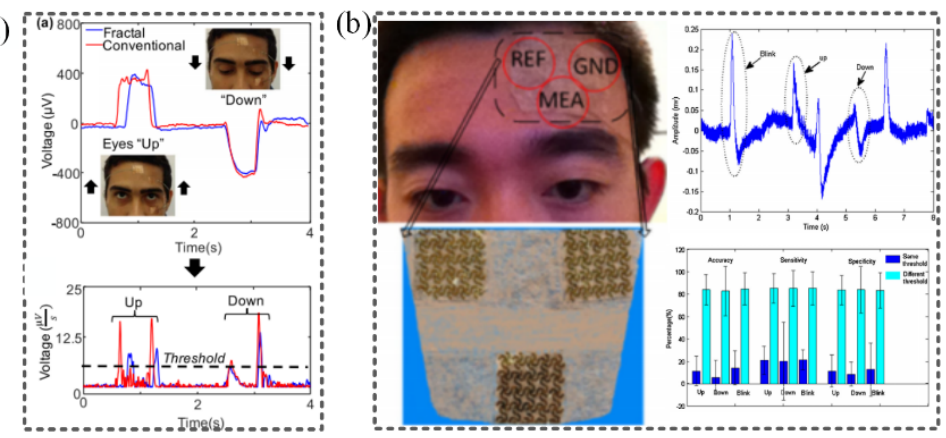

Fig.15 Stretchable EOG sensor for (a) Eye motion detection. Adapted with permission [153]. Copyright Elsevier Ltd. (b) EOG signal recordings and feature extraction with different eye motion [154]. Adapted with permission. Copyright Elsevier Ltd.

The brain-computer interface (BCI) technology is a radically new communication option for those with neuromuscular impairments that prevent them from using conventional augmentative communication methods [155]. Current BCIs use electroencephalographic (EEG) recorded at the scalp single-unit activity from cortex to control cursor movement, select letters or icons, or operate a neuroprosthesis [156]. An EEG sensor mounted on the head was designed to record EEG signals for controlling computer games shown in Fig.16(a) [14]. Fast fourier transform (FFT) method was adopted to extract features of these words [157]. As an example, dynamic time-warping pattern recognition algorithms applied to throat-based EMG data enable the control of a computer strategy game (Sokoban). The EEG sensor had the capabilities as a HMI to control the computer game [158]. The results from a BCI for a text speller were shown in Fig.16(b). The results exhibited similar patterns and amplitudes, where event-related potentials provided an additional example.
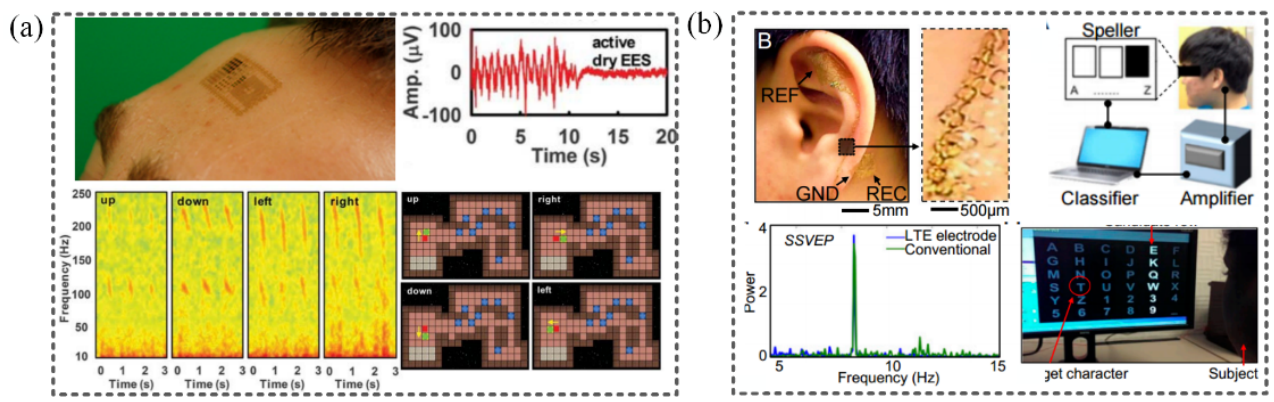

Fig.16 EEG signal via epidermal electronics for BCI application. (a) Stretchable epidermal electronics to control the computer game [14]. Adapted with permission. Copyright American Association for the Advancement of Science. (b) Interact with computer for character recognition [158]. Adapted with permission. Copyright National Academy of Sciences USA.

\section{Feedback to humans}

Stimulations based on soft tactile sensation from the physiology of the human skin to tactile sensing techniques have been studied in recent years. Several categories of stimulation to human bodies are designed, including electrotactile stimulation, sound and light stimulation, braille sheet display, and EEG-EMG 
biofeedback.

\subsection{Electrotactile stimulation}

Electrotactile stimulation evokes tactile sensations within the skin at the location of a small cutaneous electrode by passing a local electric current, through the skin to stimulate cutaneous afferent fibers. An electrocutaneous display system composed of three layers was implemented for the augmentation of skin sensation, where visual images captured by the sensor were translated into tactile information and displayed through electrotactile stimulation [159]. Integrating distributed sensing (E-skin) and stimulation (matrix electrodes), an electrotactile feedback system was proposed in Ref.[160] that helped user subjected to recognize dynamic movement patterns. It embodies closed-loop artificial devices into the user body scheme.

The human tongues could be employed as an illustrative example of HMI applications. The Tongue Display Unit (TDU, Brain Port similar to USB port) was developed for stimulating the tongue surface [161]. TDU was connected to a flexible-printed-circuit for the stimulation of the dorsal surface of a tongue as shown in Fig.17(a) [162]. The electrical stimulus to each electrode was individually controllable in real time. The block diagram of TDU comprised five 8-bit microcontrollers, electrode driver output, power and communications circuits. A type of electrotactile stimulation was designed with multiplexed using silicon nanomembrane (Si NM) diodes (Fig.17(b) [20]. The multiplexed electrotactile array was outside the freestanding finger-tube and the required voltage for sensation decreases as frequency increases, consistent with equivalent circuit models of skin impedance that involve resistors and capacitors connected in parallel. This technology could be used in applications ranging from HMIs to 'instrumented' surgical gloves [22].

(a)

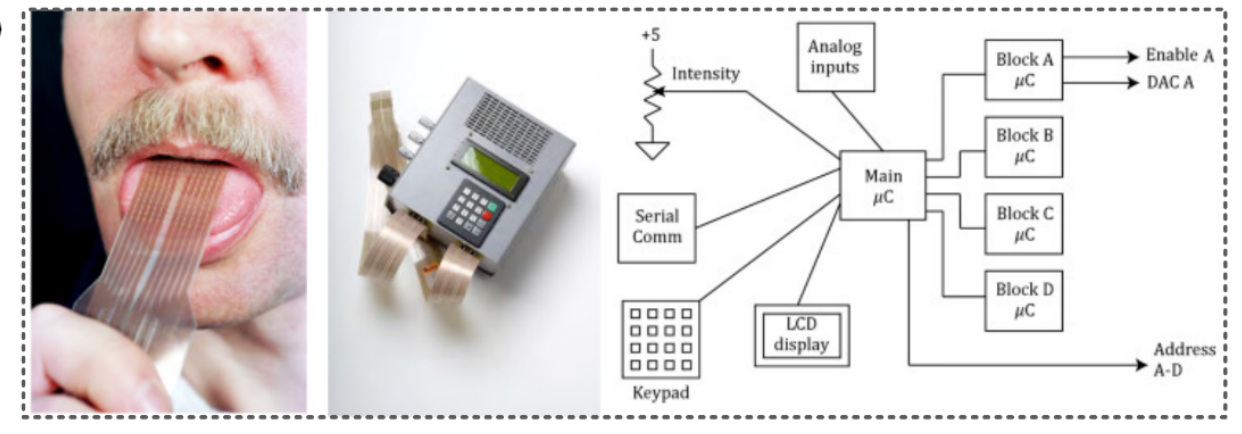

(b)

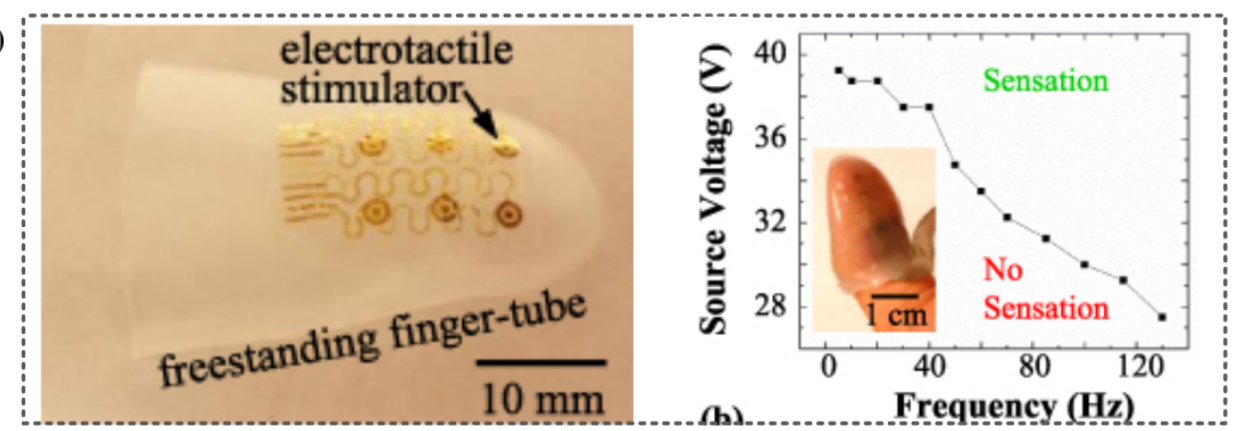

Fig.17 Electrical stimulation on tongue and fingertip for HMI. (a) Electrical stimulation to the surface of a tongue [162]. Adapted with permission. Copyright Elsevier Ltd.; (b) Electrotactile stimulation 
with electrode arrays [20]. Adapted with permission. Copyright Institute of Physics.

\subsection{Sound and light stimulation}

Sound and light stimulation to the human body is helpful to people's therapy. The visual stimulation occurs through strobe lights, while auditory stimulation was made by binaural beats. The brain stimulation by light and sound between the biofeedback techniques was pushed forward by Calomeni et al. [52]. Vieira defined the biofeedback as an immediate return of information through sensitive electronic equipment capable of capturing sensory responses, amplifying and transforming physiological signals [163]. The synthesis of brain waves fitted the definition well because the technology was able to stimulate the brain externally. Photo stimulation that highly corroborated with the possibility of brain waves could be induced through externally stimulated frequencies. The frequencies changed the state of consciousness depending on external factors such as time of stimulation, culture, and expectations of the individual [164].

Júnior et al. observed the effect of photic and auditory stimulation in the variable reaction time and skilled motor-cognitive efficiency of 20 football players [165]. Zhang designed a kind of stimulation device aiming at alleviating brain fag [166]. Meanwhile, the specific stereo signal which is expected to induce alphawave of human brains was programmed and encoded into audio files, as shown in Fig.18(a). Barnett-Cowan et al. measured the perceived timing of galvanic vestibular stimulation (GVS) relative to tactile, visual and auditory stimuli [167]. In Fig.18 (b), participants sit inside a plastic hemisphere receiving a diffused flash of light and sound stimulation respectively. A middle ear microphone was developed based on MEMS capacitive accelerometer for middle ear hearing aids as well as future fully implantable cochlear prostheses, where the sensor was interfaced with a custom-designed IC chip over a flexible substrate [168]. 


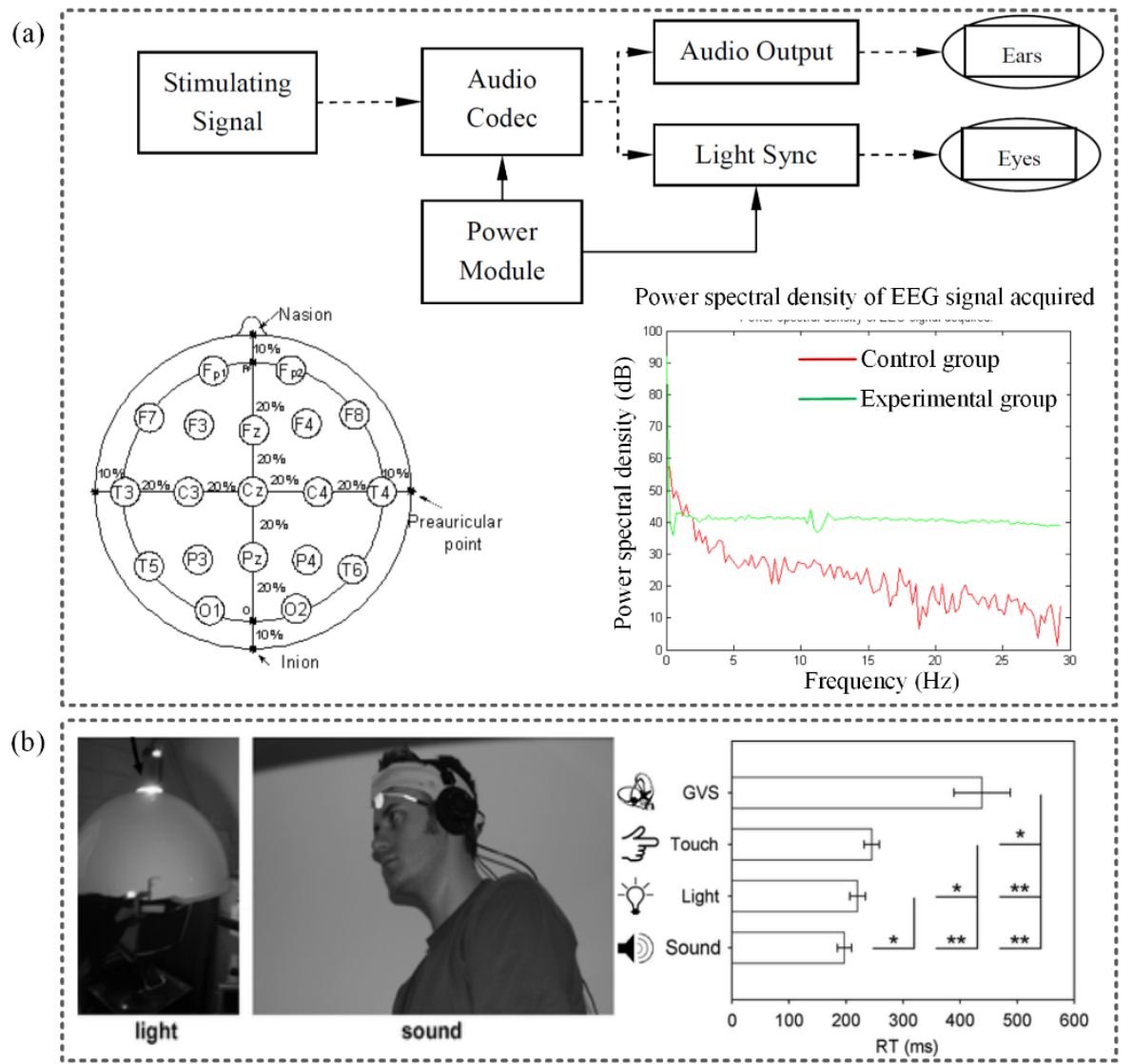

Fig.18 Sound and light stimulation for HMI application. (a) Schematic graph for stimulation to the human body [167]. Adapted with permission. Copyright Springer Verlag.; (b) Experiment setup of sound and light stimulation [168]. Adapted with permission. Copyright IEEE.

Fig.19 depicted an implantable wireless optoelectronics sensor to manipulate animal behavior via the optogenetics technologies $[169,170]$. Implantation of the wireless optoelectronics was very similar to that of traditional optic fiber ferrules and previous wireless devices. Once the region of interest was located and a hole drilled through the skull, the device could be lowered using the custom mounting fixture. The wireless optoelectronics had a red LED indicator easily visible through the skin, and used as an implantable optogenetic device for changing the behavior mode of the animal with the red light stimulation to the animal [171, 172]. A skin-inspired mechanoreceptor was developed with a flexible organic transistor circuit that transduces pressure into digital frequency signals directly, whose output was used to stimulate somatosensory neurons of mouse cortex in vitro [173]. 


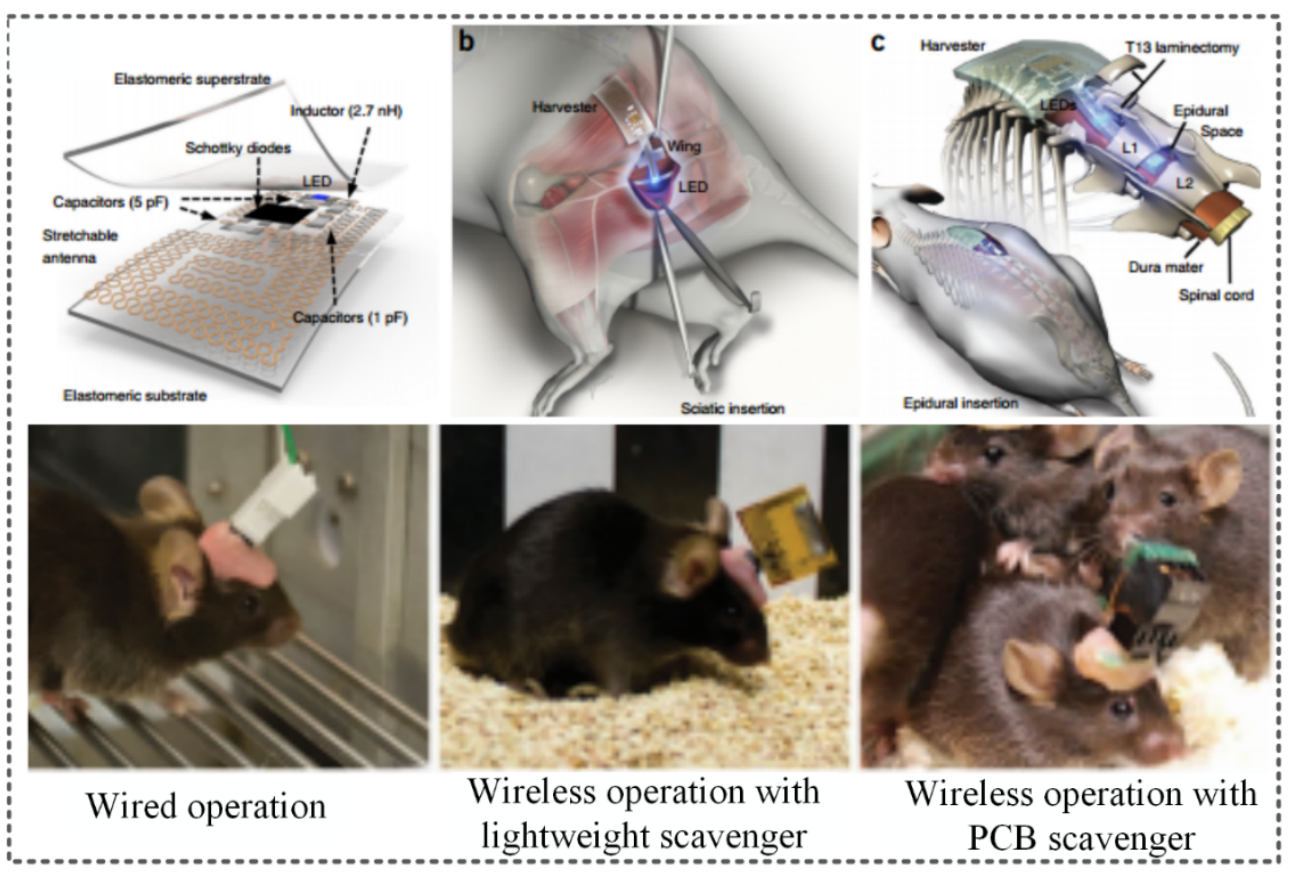

Fig.19 Wireless optoelectronics for HMI application to change animal behavior [171]. Adapted with permission. Copyright Macmillan Publishers Ltd.

\subsection{Electroactive polymer actuator for braille sheet display}

Braille sheet display is used for the visually impaired people to communicate with the outside world through touching the EAP actuators. A flexible, shock-resistant, and lightweight braille sheet display was successfully fabricated on a plastic film by integrating a plastic sheet actuator array with a high-quality organic transistor active matrix shown in Fig.20(a) [53]. The plastic MEMS actuators with organic transistor active matrices had new versatile possibilities for flexible, large-area electronic applications including tactile displays [174]. This demonstration of low-voltage control of a matrix of $\mathrm{kV}$ actuators paved the way to be used in many degrees of freedom for soft robotics, haptic displays, and flexible braille displays. When a hand touches the braille sheet display, the response information could be transformed to the subject. It provided an effective HMI for the subject to interact with the outside world via braille sheet display. Fig.20(b) depicted unprotected fingers probing the surface under the actuated condition indicating that the surfaces were electrically insulating and safe to touch [175]. $1 \mathrm{~mm}$ thick soft insulating layer on the top provided insulation from direct contact of fingers with electrodes [176]. 
(a)
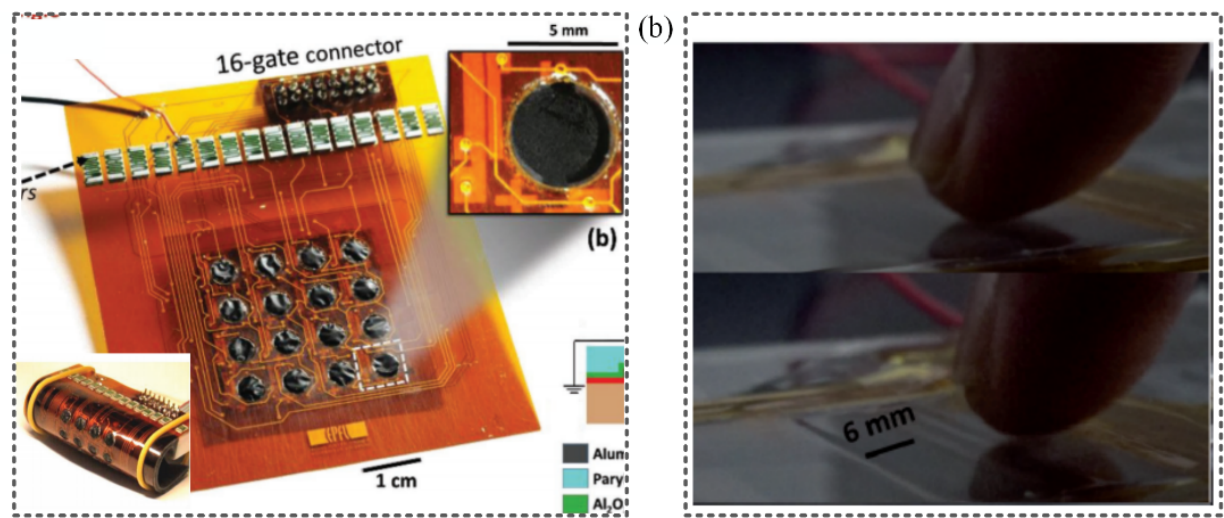

Fig.20 Stimulation to the body via EAP actuator for HMI applications. (a) Flexible EAP actuator [53].

Adapted with permission. Copyright IEEE; (b) Flexible braille sheet display [175]. Adapted with permission. Copyright IEEE.

The soft pneumatic actuators based on composites consists of flexible elastomers with embedded sheets or fiber structures which are inexpensive, simple to fabricate, light in weight, and easy to actuate [177, 178]. These soft pneumatic actuators could manipulate objects with moderate performance which they can lift loads up to 120 times their weight $[54,55]$. Soft robots were essentially more compatible for human interactions as their soft and easily deformable bodies ensured a minimal damage and load exerted to humans and environment [179]. Soft robots with ability to adapt the curved and irregular surfaces allowed overcoming the shortcomings of rigid robots [86]. Soft robotic glove for hand rehabilitation performed specific tasks for training. There were many other works on hand rehabilitation by soft exoskeleton systems [180]. Embedded with nickel nanostructured microparticles, a organic polymer performed mechanical and electrical self-healing properties at ambient conditions, and it was pressure- and flexion-sensitive, and therefore suitable for electronic skin applications in soft robotics and biomimetic prostheses [97].

\subsection{EEG-EMG biofeedback to human bodies}

EEG-EMG biofeedback training has become a useful tool for rehabilitation of impingement syndrome. It allows patients a better sense of muscle activations involved in the movement of the shoulder girdle. Contingent visual and proprioceptive feedback about the user's EEG and EMG activity is provided in the form of velocity modulation during functional task training. Participants started the real-time operation task with their paretic arm and hand relaxed in a comfortable rest position to reach one of the three targets around the workspace (Fig.21(a)-(b)), while supinating the wrist and opening their hands [181, 182]. It helped the patients accomplish complicated target task with the EEG-EMG biofeedback system and enhance the motion ability of patients.

Fig.21(c) depicted that the closed-loop system could facilitate functional neuroplastic prosthesis and eventually elicit a joint brain and muscle motor rehabilitation [183]. Its usability was validated during a realtime operation session in a healthy participant and a chronic stroke patient, showing encouraging results for 
its application to a clinical rehabilitation scenario. The calibration session was divided into an EEG screening and an EMG calibration. The EMG calibration was performed with the healthy upper limb. Information from different aspects with multiple methods about the process of EEG-EMG signals and modeling of human motion was extracted and recognized. Generally speaking, HMI based on EEG-EMG biofeedback was an effective media to establish natural connections between humans and robots.

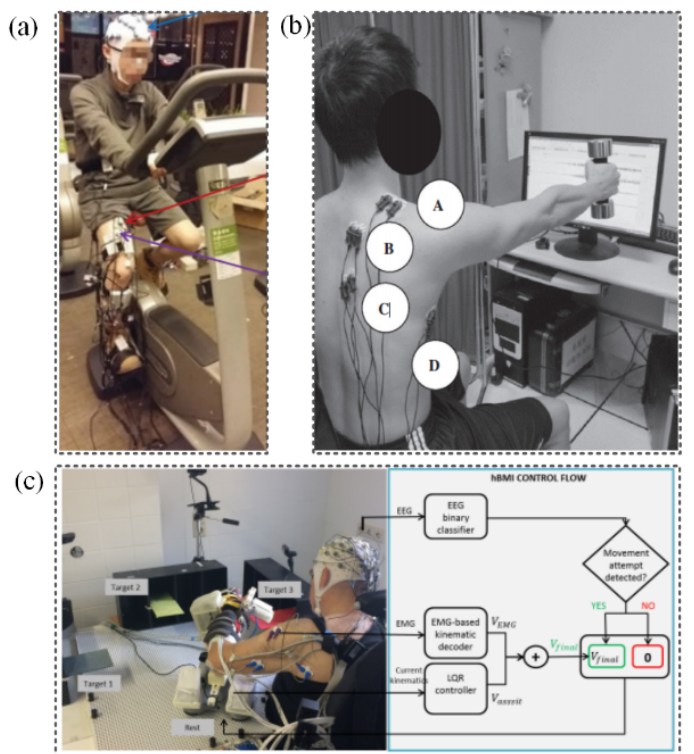

Fig.21 EEG-EMG Biofeedback training for stimulating humans.(a)-(b) Biofeedback system based on EEG-EMG electrode [181, 182] Adapted with permission. Copyright IEEE; (c) The closed-loop system based on EEG-EMG biofeedback [183]. Adapted with permission. Copyright IEEE.

Paralysis of hands and arms in persons with paraplegia or stroke reduced their dexterity, daily living skills, self-sufficiency, and vocational potential [184]. Therefore, many researchers focused on how to restore normal motor functions of upper limbs like elbow/wrist flexion and extension, hand grasp/release [185]. Since elbow motions were very important in upper limb movements, different control strategies were designed to incorporate into flexible electrical stimulation (FES) systems, inducing desired elbow flexion/extension by stimulating the specific muscles. The surface electrode array had been applied in FES systems to induce more precise stimulation control, realizing more accurate and diverse arm and hand movements (Fig.22(a)) [186].

Fig.22(b) depicted a multi-pad electrode based functional electrical stimulation system for restoration of grasping. Since patients with low-level hemiplegia retained partial volitional muscle contraction ability, researchers attempted to extract information from muscle activities that remained under voluntary control sufficient to predict appropriate stimulation levels for several paralyzed muscles in the upper extremity [187]. Considering the difficulties of producing enough joint torque and dynamic control by using FES alone, EMG- 
driven electromechanical robot system integrated with FES was developed for wrist training after stroke. The performance of the system in assisting wrist flexion/extension tracking was evaluated on five chronic stroke subjects, which showed the FES-robot assisted wrist training could enhance the hand, wrist, and elbow functions.

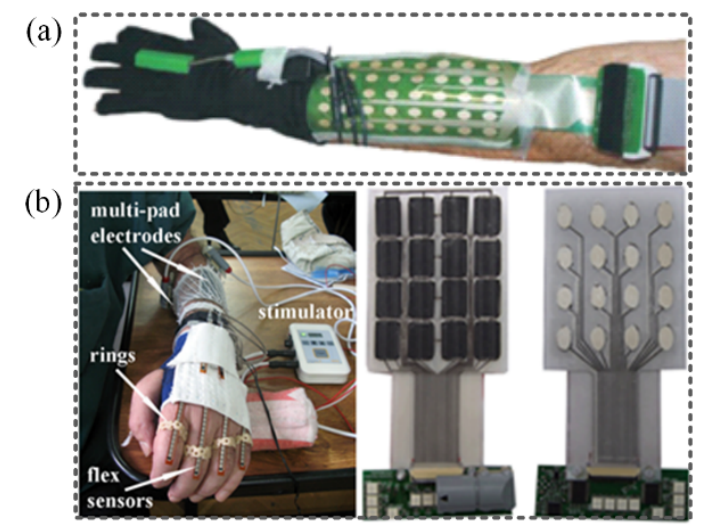

Fig.22 (a) Electrode array with data glove and electrogoniometer [186]. Adapted with permission. Copyright Elsevier Ltd.; (b) Sensor system for the assessment of the effects of stimulation [187]. Adapted with permission. Copyright Springer Verlag.

Surface FES systems can be safely used by clinicians, but accurate placements of the electrodes may be difficult and time-consuming. Fig.23(a) depicted an EMG-based implanted FES upper limb system. A great number of stimulation channels/electrodes allowed activation of greater number of muscles, which resulted in better upper limb function, for example, better grasping and releasing. And additionally FES upper limb system could provide forearm pronation and reaching by elbow extension [188]. Fig.23(b) depicted a braincontrolled FES system that had been also developed to restore grasping function following paralysis. It extracted brain neural data of monkeys to predict muscle activity, and used it to control grasping action by implanted intramuscular electrodes for stimulation of hand and forearm muscles [189]. During the early phases of rehabilitation, most persons hoped for restoration of lower limb neurological function sufficient at least to allow standing and walking. Therefore, there were researches aiming to apply FES inducing joint movements of lower limbs. In addition, FES had also been used as rehabilitation like correcting drop foot. Fig.23(c) depicted a hybrid system of exoskeletal bracing and multichannel FES to facilitate standing, walking, and stair climbing after spinal cord injury based on implanted electrodes [190]. Moreover, FES were used to induce sensory feedback, for example, tactile sensation, which was significant for non-invasive neural interface that could feed back finger-specific tactile information from the prosthetic hand to forearm amputees. 


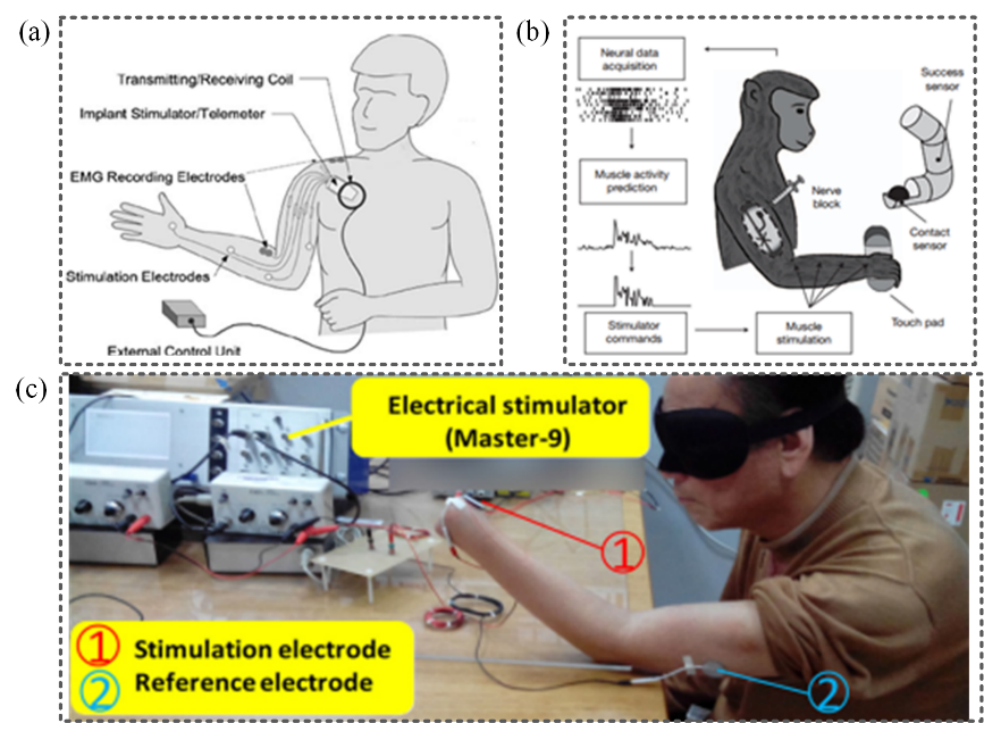

Fig.23 EMG-based implanted FES upper limb system. (a) FES upper limb system [188]. Adapted with permission. Copyright Macmillan Publishers Ltd.; (b) Brain-controlled FES system [189]. Adapted with permission. Copyright Elsevier Ltd.; (c) A hybrid system of exoskeletal bracing and multichannel FES [190]. Adapted with permission. Copyright Institute of Physics.

\section{Conclusion and Discussion}

This paper has highlighted the development of soft HMIs based on flexible and stretchable electronics technologies, including flexible/stretchable tactile sensors, motion sensors, biological sensors, and stimulation feedback to human bodies. Soft HMIs will play a key role in human-centered applications including robotics, sports, automobiles, textiles, and many other fields. Flexible/stretchable motion sensors are critically important for the robot and humans to accomplish complicated and dynamic tasks. Electrophysiology sensors have been developed as HMIs to EMG, ECG, and EOG signal recordings. The flexible electronic devices can stimulate human bodies for enhancing manual ability. Highly integrated electronics for the detection of multiple stimuli are the subject of many investigations. With the aid of newly emerging technologies, such as wireless sensor networks and ultrathin sensors, these efforts have received substantial attention in the field of health monitoring, medical implant services, and HMIs.

Recent developments in material science, nanotechnology, micro-/nano-fabrication techniques can improve HMI technologies in terms of performance, reliability, and miniaturization. (1) Conformal HMIs are designed and fabricated with flexible and stretchable electronics to provide a more friendly interface between human bodies and machines. Artificial skins with tactile sensors designed to mimic human skin in structural, functional, physiological and mechanical attributes, are promising in HMI applications. Flexible electronic devices have been used in many applications in recent tactile sensing technologies for their biocompatibility 
and excellent mechanical properties. (2) Soft tactile and bio-potential sensors are developed to improve human body rehabilitation as one type of HMIs, where flexile and stretchable electronics can be connected to a human nerve as part of human body. Stimulations to human bodies are generated by feedback information from the soft electronic devices integrated with soft tactile sensors or bio-potential sensors. (3) Soft HMIs promote interdisciplinary researches, such as flexible hybrid electronic manufacturing in the range of physics, material science, chemistry, informatics, manufacturing and so on. It would give an effective solution to fully flexible systems with soft sensors, soft printed circuit boards, and soft processors for improving the biocompatibility with human bodies.

However, there still exists some difficulties for the development of soft HMIs in the field of service robot and healthcare: (1) High manufacturing cost of soft HMIs among the critical issues to be considered in development. The low-cost materials and simple fabrication processes are desired to reduce cost. In a similar way, devices with low-power consumption or self-powering ability are worthy of in-depth studies. (2) More advanced intelligent sensing and congestive technologies are needed. Very large information and data must be exchanged between the human and machine for interacting more effectively. New algorithms are required for recognizing sensor signals to improve the communication and interaction efficiency, by mimicking real human skin which can adjust and provide feedback in real time according to the different types of external stimuli via the peripheral nervous systems. Future HMIs will also intelligently respond to variations in the external environment based on novel information transmission technology. (3) Coexistence safety could be a problem between the humans and machines. Multi-sensory and intelligent electronic devices should be thoroughly studied for future HMI applications, such as Tri-Co Robots. This provides an effective means for humans to interact with robots or machines in a similar way as human-to-human interactions. So soft HMIs provide a promising solution for a safe and friendly interaction between humans and robots.

\section{Acknowledgement}

The authors would like to acknowledge supports from the National Natural Science Foundation of China (51635007, 91748113, 51575412), Program for HUST Academic Frontier Youth Team, Special Project of Technology Innovation of Hubei Province. (2017AAA002), and State Key Lab of Digital Manufacturing Equipment \& Technology, China (DMETKF2017003). The authors would like to thank Flexible Electronics Manufacturing Laboratory in Comprehensive Experiment Center for Advanced Manufacturing and Equipment Technology. 


\section{References}

[1] H. Liu, Z. Ju, X. Ji, C. S. Chan, and M. Khoury, Human Motion Sensing and Recognition: Springer Berlin Heidelberg, 2017.

[2] H. Yu, S. Huang, G. Chen, Y. Pan, and Z. Guo, "Human-Robot Interaction Control of Rehabilitation Robots With Series Elastic Actuators," IEEE Transactions on Robotics, vol. 31, pp. 1089-1100, 2015.

[3] J. Yi, J. Ueda, and X. Zhu, "Introduction to the focused section on intelligent robotics for rehabilitation and human assistance," International Journal of Intelligent Robotics and Applications, vol. 1, pp. 3-5, 2017/02/01 2017.

[4] S. Uchida, A. Mori, R. Kurazume, T. Rin-Ichiro, T. Hasegawa, and H. Sakoe, "Early Recognition and Prediction of Gestures for Proactive Human-Machine Interface," Technical Report of Ieice Prmu, vol. 104, pp. 7-12, 2004.

[5] C. Brown, M. Cowell, C. A. Plont, H. Hahn, and D. Mascareñas, "A Vibro-Haptic Human-Machine Interface for Structural Health Monitoring Applications," Structural Health Monitoring, vol. 13, pp. 671-685, 2014.

[6] J. He, D. Zhang, X. Sheng, S. Li, and X. Zhu, "Invariant Surface EMG Feature Against Varying Contraction Level for Myoelectric Control Based on Muscle Coordination," IEEE Journal of Biomedical \& Health Informatics, vol. 19, p. 874, 2015.

[7] T. I. Kim, J. G. McCall, Y. H. Jung, X. Huang, E. R. Siuda, Y. Li, J. Song, Y. M. Song, H. A. Pao, R. H. Kim, C. Lu, S. D. Lee, I. S. Song, G. Shin, R. Al-Hasani, S. Kim, M. P. Tan, Y. Huang, F. G. Omenetto, J. A. Rogers, and M. R. Bruchas, "Injectable, cellular-scale optoelectronics with applications for wireless optogenetics," Science, vol. 340, pp. 211-6, Apr 122013.

[8] H. Liu, Z. Ju, X. Ji, C. S. Chan, and M. Khoury, "Human Hand Motion Analysis with Multisensory Information," IEEE/ASME Transactions on Mechatronics, vol. 19, pp. 456-466, 2014.

[9] X. Wang, L. Dong, H. Zhang, R. Yu, C. Pan, and Z. L. Wang, "Recent Progress in Electronic Skin," Advanced Science, vol. 2, 2015.

[10] M. L. Hammock, A. Chortos, B. C. Tee, J. B. Tok, and Z. Bao, "25th anniversary article: The evolution of electronic skin (e-skin): a brief history, design considerations, and recent progress," Adv Mater, vol. 25, pp. 5997-6038, Nov 132013.

[11] J. A. Rogers, T. Someya, and Y. Huang, "Materials and mechanics for stretchable electronics," Science, vol. 327, pp. 1603-7, Mar 262010.

[12] Y. Huang, Y. Ding, J. Bian, Y. Su, J. Zhou, Y. Duan, and Z. Yin, "Hyper-stretchable self-powered sensors based on electrohydrodynamically printed, self-similar piezoelectric nano/microfibers," Nano Energy, vol. 40, pp. 432-439, 2017/10/01/2017.

[13] D. J. Lipomi, M. Vosgueritchian, B. C. Tee, S. L. Hellstrom, J. A. Lee, C. H. Fox, and Z. Bao, "Skin-like pressure and strain sensors based on transparent elastic films of carbon nanotubes," Nat Nanotechnol, vol. 6, pp. 788-92, Dec 2011.

[14] D. H. Kim, N. Lu, R. Ma, Y. S. Kim, R. H. Kim, S. Wang, J. Wu, S. M. Won, H. Tao, A. Islam, K. J. Yu, T. I. Kim, R. Chowdhury, M. Ying, L. Xu, M. Li, H. J. Chung, H. Keum, M. McCormick, P. Liu, Y. W. Zhang, F. G. Omenetto, Y. Huang, T. Coleman, and J. A. Rogers, "Epidermal electronics," Science, vol. 333, pp. 838-43, Aug 122011.

[15] H. Ding, X. Yang, N. Zheng, M. Li, Y. Lai, and H. Wu, "Tri-Co Robot: a Chinese robotic research initiative for enhanced robot interaction capabilities," National Science Review, pp. nwx148-nwx148, 2017.

[16] S. C. Mannsfeld, B. C. Tee, R. M. Stoltenberg, C. V. Chen, S. Barman, B. V. Muir, A. N. Sokolov, C. Reese, and Z. Bao, "Highly sensitive flexible pressure sensors with microstructured rubber dielectric layers," Nat Mater, vol. 9, pp. 859-64, Oct 2010.

[17] B. C. Tee, C. Wang, R. Allen, and Z. Bao, "An electrically and mechanically self-healing composite with pressure- and flexion-sensitive properties for electronic skin applications," Nat Nanotechnol, vol. 7, pp. 825-32, Dec 2012.

[18] S. Lim, D. Son, J. Kim, Y. B. Lee, J.-K. Song, S. Choi, D. J. Lee, J. H. Kim, M. Lee, T. Hyeon, and D.-H. Kim, "Transparent and Stretchable Interactive Human Machine Interface Based on Patterned Graphene Heterostructures," 
Advanced Functional Materials, vol. 25, pp. 375-383, 2015.

[19] J. Y. Sun, C. Keplinger, G. M. Whitesides, and Z. Suo, "Ionic skin," Advanced Materials, vol. 26, pp. 7608-7614, 2015.

[20] M. Ying, A. P. Bonifas, N. Lu, Y. Su, R. Li, H. Cheng, A. Ameen, Y. Huang, and J. A. Rogers, "Silicon nanomembranes for fingertip electronics," Nanotechnology, vol. 23, p. 344004, Aug 312012.

[21] X. Xiao, L. Yuan, J. Zhong, T. Ding, Y. Liu, Z. Cai, Y. Rong, H. Han, J. Zhou, and Z. L. Wang, "High-strain sensors based on ZnO nanowire/polystyrene hybridized flexible films," Adv Mater, vol. 23, pp. 5440-4, Dec 12011.

[22] D. H. Kim, S. Wang, H. Keum, R. Ghaffari, Y. S. Kim, H. Tao, B. Panilaitis, M. Li, Z. Kang, F. Omenetto, Y. Huang, and J. A. Rogers, "Thin, flexible sensors and actuators as 'instrumented' surgical sutures for targeted wound monitoring and therapy," Small, vol. 8, pp. 3263-8, Nov 52012.

[23] X. Huang, Y. Liu, H. Cheng, W. J. Shin, J. A. Fan, Z. Liu, C. J. Lu, G. W. Kong, K. Chen, and D. Patnaik, "Materials and Designs for Wireless Epidermal Sensors of Hydration and Strain," Advanced Functional Materials, vol. 24, pp. 38463854, 2014.

[24] S. H. Jeong, S. Zhang, K. Hjort, J. Hilborn, and Z. Wu, "PDMS - based elastomer tuned soft, stretchable, and sticky for epidermal electronics," Advanced Materials, 2016.

[25] X. Ji, H. Liu, Y. Li, and D. Brown, "Visual-Based View-Invariant Human Motion Analysis: A Review," vol. 5177, pp. 741-748, 2008.

[26] X. Ji and H. Liu, "Advances in View-Invariant Human Motion Analysis: A Review," IEEE Transactions on Systems Man \& Cybernetics Part C, vol. 40, pp. 13-24, 2009.

[27] W. Guo, X. Sheng, H. Liu, and X. Zhu, "Toward an Enhanced Human-Machine Interface for Upper-Limb Prosthesis Control With Combined EMG and NIRS Signals," IEEE Transactions on Human-Machine Systems, vol. 47, pp. 564-575, 2017.

[28] O. Abdeljaber, O. Avci, and D. J. Inman, "Active vibration control of flexible cantilever plates using piezoelectric materials and artificial neural networks," Journal of Sound \& Vibration, vol. 363, 2015.

[29] A. H. Rajabi, M. Jaffe, and T. L. Arinzeh, "Piezoelectric materials for tissue regeneration: A review," Acta Biomaterialia, vol. 24, pp. 12-23, 2015.

[30] Shriver, Stefanie, Toth, Arthur, Zhu, Xiaojin, Rudnicky, Alex, Rosenfeld, and Roni, "A unified design for human-machine voice interaction," pp. 247--248, 2001.

[31] B. Wang, Z. Li, W. Ye, and Q. Xie, "Development of human-machine interface for teleoperation of a mobile manipulator," International Journal of Control Automation \& Systems, vol. 10, pp. 1225-1231, 2012.

[32] M. Watanabe, Y. Yamamoto, K. Nakakoji, and Y. Koike, "Multi-DOF motion controller by EMG based human-machine interface using operator's joint torque and stiffness," Ieice Technical Report Neurocomputing, vol. 106, pp. 91-96, 2006.

[33] B. O Flynn, J. Sanchez, P. Angove, J. Connolly, J. Condell, and K. Curran, "Novel Smart Sensor Glove for Arthritis Rehabilitation," pp. 1-6, 2014.

[34] N. Lu, C. Lu, S. Yang, and J. Rogers, "Highly sensitive skin - mountable strain gauges based entirely on elastomers," Advanced Functional Materials, vol. 22, pp. 4044-4050, 2012.

[35] Y. Huang, Y. Wang, L. Xiao, H. Liu, W. Dong, and Z. Yin, "Microfluidic serpentine antennas with designed mechanical tunability," Lab on a Chip, vol. 14, pp. 4205-4212, 2014.

[36] J. Park, Y. Na, G. Gu, and J. Kim, "Flexible insole ground reaction force measurement shoes for jumping and running," in IEEE International Conference on Biomedical Robotics and Biomechatronics, 2016, pp. 1062-1067.

[37] M. Domazet, "Human-machine interaction based on EMG measurements," 2016.

[38] Q. Han, X. Chen, K. Tang, and P. Li, "A non-contact human-computer interaction application design based on electrostatic current of human body," International Journal of Computer Applications in Technology, vol. 53, pp. 23-31, 2016.

[39] S. Saproo, V. Shih, D. C. Jangraw, and P. Sajda, "Neural mechanisms underlying catastrophic failure in human-machine interaction during aerial navigation," Journal of Neural Engineering, vol. 13, p. 066005, 2016.

[40] Löhrer, MarioLemm, JacquelineGloy, YvesSimonGries, and Thomas, "Adaptive supporting systems for a competence- 
enhancing human-machine interaction in new production processes," Nature, vol. 205, pp. 773-6, 2015.

[41] S. Mavadati, "Spontaneous Facial Behavior Computing in Human Machine Interaction with Applications in Autism Treatment," Dissertations \& Theses - Gradworks, 2015.

[42] J. A. Rogers, "Materials science. A clear advance in soft actuators," Science, vol. 341, pp. 968-9, Aug 302013.

[43] T. George, M. S. Islam, A. K. Dutta, D.-H. Kim, J. Lee, and M. Park, "High performance bio-integrated devices," vol. 9083, p. $90831 \mathrm{H}, 2014$.

[44] Y. Hu and Z. L. Wang, "Recent progress in piezoelectric nanogenerators as a sustainable power source in self-powered systems and active sensors," Nano Energy, vol. 14, pp. 3-14, 2015.

[45] W. Gao, S. Emaminejad, H. Y. Y. Nyein, S. Challa, K. Chen, A. Peck, H. M. Fahad, H. Ota, H. Shiraki, D. Kiriya, D. H. Lien, G. A. Brooks, R. W. Davis, and A. Javey, "Fully integrated wearable sensor arrays for multiplexed in situ perspiration analysis," Nature, vol. 529, pp. 509-514, Jan 282016.

[46] X. Huang, Y. Liu, G. W. Kong, J. H. Seo, Y. Ma, K.-I. Jang, J. A. Fan, S. Mao, Q. Chen, D. Li, H. Liu, C. Wang, D. Patnaik, L. Tian, G. A. Salvatore, X. Feng, Z. Ma, Y. Huang, and J. A. Rogers, "Epidermal radio frequency electronics for wireless power transfer," Microsystems \& Nanoengineering, vol. 2, p. 16052, 2016.

[47] J. W. Lee, R. Xu, S. Lee, K. I. Jang, Y. Yang, A. Banks, K. J. Yu, J. Kim, S. Xu, S. Ma, S. W. Jang, P. Won, Y. Li, B. H. Kim, J. Y. Choe, S. Huh, Y. H. Kwon, Y. Huang, U. Paik, and J. A. Rogers, "Soft, thin skin-mounted power management systems and their use in wireless thermography," Proc Natl Acad Sci U S A, vol. 113, pp. 6131-6, May 312016.

[48] B. Xu, A. Akhtar, Y. Liu, H. Chen, W.-H. Yeo, S. Park, II, B. Boyce, H. Kim, J. Yu, H.-Y. Lai, S. Jung, Y. Zhou, J. Kim, S. Cho, Y. Huang, T. Bretl, and J. A. Rogers, "An Epidermal Stimulation and Sensing Platform for Sensorimotor Prosthetic Control, Management of Lower Back Exertion, and Electrical Muscle Activation," Advanced Materials, vol. 28, pp. 4462-4471, 2016.

[49] X. He, Y. Zi, H. Yu, S. L. Zhang, J. Wang, W. Ding, H. Zou, W. Zhang, C. Lu, and Z. L. Wang, "An ultrathin paper-based self-powered system for portable electronics and wireless human-machine interaction," Nano Energy, vol. 39, pp. 328$336,2017$.

[50] L. Haviv, H. Friedman, U. Bierman, I. Glass, A. Plotkin, A. Weissbrod, S. Shushan, V. Bluvshtein, E. Aidinoff, N. Sobel, and A. Catz, "Using a Sniff Controller to Self-Trigger Abdominal Functional Electrical Stimulation for Assisted Coughing Following Cervical Spinal Cord Lesions," IEEE Transactions on Neural Systems and Rehabilitation Engineering, vol. 25, pp. 1461-1471, 2017.

[51] A. K. Martin, M. Meinzer, R. Lindenberg, M. M. Sieg, L. Nachtigall, and A. Flöel, "Effects of Transcranial Direct Current Stimulation on Neural Networks Structure in Young and Older Adults," Journal of Cognitive Neuroscience, vol. 29, pp. 1817-1828, 2017.

[52] M. R. Calomeni, J. A. Rocha, A. P. Silva, L. H. Ribeiro, L. Marques, M. A. Siza, and V. F. Silva, "Brain stimulation used as biofeedback training for recovery of motor functions deteriorated by stroke," Arquivos de neuro-psiquiatria, vol. 71, pp. 159-64, 2013.

[53] A. Marette, A. Poulin, N. Besse, S. Rosset, D. Briand, and H. Shea, "Flexible Zinc-Tin Oxide Thin Film Transistors Operating at $1 \mathrm{kV}$ for Integrated Switching of Dielectric Elastomer Actuators Arrays," Advanced Materials, vol. $29,2017$.

[54] R. V. Martinez, C. R. Fish, X. Chen, and G. M. Whitesides, "Elastomeric Origami: Programmable Paper - Elastomer Composites as Pneumatic Actuators," Advanced Functional Materials, vol. 22, pp. 1376-1384, 2012.

[55] J. Bishop-Moser and S. Kota, "Design and Modeling of Generalized Fiber-Reinforced Pneumatic Soft Actuators," IEEE Transactions on Robotics, vol. 31, pp. 536-545, 2017.

[56] N. Ranasinghe and Y. L. Do, "Digital Lollipop: Studying Electrical Stimulation on the Human Tongue to Simulate Taste Sensations," Acm Transactions on Multimedia Computing Communications \& Applications, vol. 13, p. 5, 2016.

[57] S. Löffler, B. Libberton, and A. Richter-Dahlfors, "Organic Bioelectronic Tools for Biomedical Applications," Electronics, vol. 4, pp. 879-908, 2015.

[58] Y. Zou, H. Wan, X. Zhang, H. Da, and P. Wang, "Electronic Nose and Electronic Tongue," 2015. 
[59] J. M. Nightingale and R. W. Todd, "An Adaptively-Controlled Prosthetic Hand," ARCHIVE Engineering in Medicine 1971-1988 (vols 1-17), vol. 1, pp. 3-6, 1971.

[60] G. Beni, S. Hackwood, and W. S. Trimmer, "High-precision robot system for inspection and testing of electronic devices," in IEEE International Conference on Robotics and Automation. Proceedings, 1984, pp. 428-441.

[61] P. Dario, D. D. Rossi, C. Giannotti, F. Vivaldi, and P. C. Pinotti, "Ferroelectric polymer tactile sensors for prostheses," Ferroelectrics, vol. 60, pp. 199-214, 1984.

[62] R. S. Johansson, "Tactile sensibility in the human hand: receptive field characteristics of mechanoreceptive units in the glabrous skin area," Journal of Physiology, vol. 281, p. 101, 1978.

[63] T. A. Chase and R. C. Luo, "A thin-film flexible capacitive tactile normal/shear force array sensor," in IEEE IECON International Conference on Industrial Electronics, Control, and Instrumentation, 1995, pp. 1196-1201 vol.2.

[64] S. G. Tzafestas, "Fuzzy systems and fuzzy expert control: An overview," Knowledge Engineering Review, vol. 9, pp. 229268, 1994.

[65] S. Somarajan, S. Cassilly, C. Obioha, L. A. Bradshaw, and W. O. Richards, "Noninvasive biomagnetic detection of isolated ischemic bowel segments," IEEE Transactions on Biomedical Engineering, vol. 60, pp. 1677-1684, 2013.

[66] T. Someya, T. Sekitani, S. Iba, Y. Kato, H. Kawaguchi, and T. Sakurai, "A large-area, flexible pressure sensor matrix with organic field-effect transistors for artificial skin applications," Proc Natl Acad Sci U S A, vol. 101, pp. 9966-70, Jul 6 2004.

[67] T. Sekitani, H. Nakajima, H. Maeda, T. Fukushima, T. Aida, K. Hata, and T. Someya, "Stretchable active-matrix organic light-emitting diode display using printable elastic conductors," Nat Mater, vol. 8, pp. 494-9, Jun 2009.

[68] T. Sekitani, T. Yokota, U. Zschieschang, H. Klauk, S. Bauer, K. Takeuchi, M. Takamiya, T. Sakurai, and T. Someya, "Organic nonvolatile memory transistors for flexible sensor arrays," Science, vol. 326, pp. 1516-9, Dec 112009.

[69] H. C. Ko, M. P. Stoykovich, J. Song, V. Malyarchuk, W. M. Choi, C. J. Yu, G. J. Rd, J. Xiao, S. Wang, and Y. Huang, "A hemispherical electronic eye camera based on compressible silicon optoelectronics," Nature, vol. 454, p. 748, 2008.

[70] W. Wu and Z. L. Wang, "Piezotronics and piezo-phototronics for adaptive electronics and optoelectronics," Nature Reviews Materials, vol. 1, p. 16031, 2016.

[71] K. Takei, T. Takahashi, J. C. Ho, H. Ko, A. G. Gillies, P. W. Leu, R. S. Fearing, and A. Javey, "Nanowire active-matrix circuitry for low-voltage macroscale artificial skin," Nat Mater, vol. 9, pp. 821-6, Oct 2010.

[72] X. Qian, M. Su, F. Li, and Y. Song, "Research Progress in Flexible Wearable Electronic Sensors," Acta Chimica Sinica, vol. 74, p. 565, 2016.

[73] J. A. Fan, W. H. Yeo, Y. Su, Y. Hattori, W. Lee, S. Y. Jung, Y. Zhang, Z. Liu, H. Cheng, L. Falgout, M. Bajema, T. Coleman, D. Gregoire, R. J. Larsen, Y. Huang, and J. A. Rogers, "Fractal design concepts for stretchable electronics," Nat Commun, vol. 5, p. 3266, 2014.

[74] J. W. Jeong, W. H. Yeo, A. Akhtar, J. J. Norton, Y. J. Kwack, S. Li, S. Y. Jung, Y. Su, W. Lee, J. Xia, H. Cheng, Y. Huang, W. S. Choi, T. Bretl, and J. A. Rogers, "Materials and optimized designs for human-machine interfaces via epidermal electronics," Adv Mater, vol. 25, pp. 6839-46, Dec 172013.

[75] J. Kim, M. Lee, H. J. Shim, R. Ghaffari, H. R. Cho, D. Son, Y. H. Jung, M. Soh, C. Choi, S. Jung, K. Chu, D. Jeon, S. T. Lee, J. H. Kim, S. H. Choi, T. Hyeon, and D. H. Kim, "Stretchable silicon nanoribbon electronics for skin prosthesis," Nat Commun, vol. 5, p. 5747, 2014.

[76] Y. Liu, J. J. Norton, R. Qazi, Z. Zou, K. R. Ammann, H. Liu, L. Yan, P. L. Tran, K. I. Jang, and J. W. Lee, "Epidermal mechano-acoustic sensing electronics for cardiovascular diagnostics and human-machine interfaces," Science Advances, vol. 2, pp. e1601185-e1601185, 2016.

[77] K. C. Pradel, W. Wu, Y. Ding, and Z. L. Wang, "Solution-derived ZnO homojunction nanowire films on wearable substrates for energy conversion and self-powered gesture recognition," Nano Lett, vol. 14, pp. 6897-905, Dec 102014.

[78] J. Kim, M. Lee, J. S. Rhim, P. Wang, N. Lu, and D. H. Kim, "Next-generation flexible neural and cardiac electrode arrays," Biomedical Engineering Letters, vol. 4, pp. 95-108, 2014. 
[79] Y. Pang, J. Li, T. Zhou, Z. Yang, J. Luo, L. Zhang, G. Dong, C. Zhang, and Z. L. Wang, "Flexible transparent tribotronic transistor for active modulation of conventional electronics," Nano Energy, vol. 31, pp. 533-540, 2017.

[80] B. Xu, A. Akhtar, Y. Liu, H. Chen, W. H. Yeo, S. I. Park, B. Boyce, H. Kim, J. Yu, H. Y. Lai, S. Jung, Y. Zhou, J. Kim, S. Cho, Y. Huang, T. Bretl, and J. A. Rogers, "An Epidermal Stimulation and Sensing Platform for Sensorimotor Prosthetic Control, Management of Lower Back Exertion, and Electrical Muscle Activation," Adv Mater, vol. 28, pp. 4462-71, Jun 2016.

[81] D.-H. Kim and J. A. Rogers, "Stretchable Electronics: Materials Strategies and Devices," Advanced Materials, vol. 20 , pp. 4887-4892, 2008.

[82] B. D. Argall and A. G. Billard, "A survey of Tactile Human-Robot Interactions," Robotics and Autonomous Systems, vol. 58, pp. 1159-1176, 2010.

[83] G. Y. Gu, J. Zhu, L. M. Zhu, and X. Zhu, "A survey on dielectric elastomer actuators for soft robots," Bioinspir Biomim, vol. 12, p. 011003, Jan 232017.

[84] C. Lee, M. Kim, Y. J. Kim, N. Hong, S. Ryu, H. J. Kim, and S. Kim, "Soft robot review," International Journal of Control, Automation and Systems, vol. 15, pp. 3-15, 2017.

[85] S. Zhao, J. Li, D. Cao, G. Zhang, J. Li, K. Li, Y. Yang, W. Wang, Y. Jin, R. Sun, and C. P. Wong, "Recent Advancements in Flexible and Stretchable Electrodes for Electromechanical Sensors: Strategies, Materials, and Features," ACS Appl Mater Interfaces, vol. 9, pp. 12147-12164, Apr 122017.

[86] P. Polygerinos, N. Correll, S. A. Morin, B. Mosadegh, C. D. Onal, K. Petersen, M. Cianchetti, M. T. Tolley, and R. F. Shepherd, "Soft Robotics: Review of Fluid - Driven Intrinsically Soft Devices; Manufacturing, Sensing, Control, and Applications in Human - Robot Interaction," Advanced Engineering Materials, vol. 19, 2017.

[87] Y. Xue, Z. Ju, K. Xiang, J. Chen, and H. Liu, "Multimodal Human Hand Motion Sensing and Analysis -A Review," IEEE Transactions on Cognitive \& Developmental Systems, vol. PP, pp. 1-1, 2018.

[88] N. Lu and D.-H. Kim, "Flexible and Stretchable Electronics Paving the Way for Soft Robotics," Soft Robotics, vol. 1, pp. 53-62, 2014.

[89] X. Wang, L. Dong, H. Zhang, R. Yu, C. Pan, and Z. L. Wang, "Recent Progress in Electronic Skin," Advanced Science, vol. 2, p. 1500169, 2015.

[90] V. C. Sundar, J. Zaumseil, V. Podzorov, E. Menard, R. L. Willett, T. Someya, M. E. Gershenson, and J. A. Rogers, "Elastomeric transistor stamps: reversible probing of charge transport in organic crystals," Science, vol. 303, p. 1644, 2004.

[91] T. Cheng, Y.-Z. Zhang, J.-D. Zhang, W.-Y. Lai, and W. Huang, "High-performance free-standing PEDOT: PSS electrodes for flexible and transparent all-solid-state supercapacitors," Journal of Materials Chemistry A, vol. 4, pp. 10493-10499, 2016.

[92] Y. Yu, T. Nguyen, P. Tathireddy, D. J. Young, and S. Roundy, "Wireless hydrogel-based glucose sensor for future implantable applications," in SENSORS, 2016 IEEE, 2016, pp. 1-3.

[93] M. Ding, T. Matsubara, Y. Funaki, R. Ikeura, T. Mukai, and T. Ogasawara, "Generation of comfortable lifting motion for a human transfer assistant robot," International Journal of Intelligent Robotics and Applications, vol. 1, pp. 74-85, 2017/02/01 2017.

[94] B. Chen, Y. Bai, F. Xiang, J. Y. Sun, Y. Mei Chen, H. Wang, J. Zhou, and Z. Suo, "Stretchable and transparent hydrogels as soft conductors for dielectric elastomer actuators," Journal of Polymer Science Part B Polymer Physics, vol. 52, pp. 1055-1060, 2014.

[95] C. H. Liu, W. Chen, W. Su, and C. N. Sun, "Numerical and experimental analysis of the automated demolding process for PDMS microfluidic devices with high-aspect ratio micropillars," International Journal of Advanced Manufacturing Technology, vol. 80, pp. 401-409, 2015.

[96] T. Yabuta, E. P. Bescher, J. D. Mackenzie, K. Tsuru, S. Hayakawa, and A. Osaka, "Synthesis of PDMS-Based Porous Materials for Biomedical Applications," Journal of Sol-Gel Science and Technology, vol. 26, pp. 1219-1222, 2003. 
[97] C. K. Tee, C. Wang, R. Allen, and Z. Bao, "An electrically and mechanically self-healing composite with pressure- and flexion-sensitive properties for electronic skin applications," Nature Nanotechnology, vol. 7, pp. 825-32, 2012.

[98] Y. Huang, Z. Yin, and Y. Xiong, "Thermomechanical analysis of thin films on temperature-dependent elastomeric substrates in flexible heterogeneous electronics," Thin Solid Films, vol. 518, pp. 1698-1702, 1/1/2010.

[99] Y. Su, X. Ping, K. J. Yu, J. W. Lee, J. A. Fan, B. Wang, M. Li, R. Li, D. V. Harburg, and Y. Huang, "In-Plane Deformation Mechanics for Highly Stretchable Electronics," Advanced Materials, vol. 29, 2017.

[100] M. Gonzalez, F. Axisa, M. V. Bulcke, D. Brosteaux, B. Vandevelde, and J. Vanfleteren, "Design of metal interconnects for stretchable electronic circuits," Microelectronics Reliability, vol. 48, pp. 825-832, 2008.

[101] Y. Zhang, H. Fu, Y. Su, S. Xu, H. Cheng, J. A. Fan, K.-C. Hwang, J. A. Rogers, and Y. Huang, "Mechanics of ultrastretchable self-similar serpentine interconnects," Acta Materialia, vol. 61, pp. 7816-7827, 2013.

[102] Y. Zhang, S. Xu, H. Fu, J. Lee, J. Su, K. C. Hwang, J. A. Rogers, and Y. Huang, "Buckling in serpentine microstructures and applications in elastomer-supported ultra-stretchable electronics with high areal coverage," Soft Matter, vol. 9, pp. 8062-8070, 2013.

[103] R. Li, M. Li, Y. Su, J. Song, and X. Ni, "An analytical mechanics model for the island-bridge structure of stretchable electronics," Soft Matter, vol. 9, p. 8476, 2013.

[104] Y. W. Su, S. D. Wang, Y. A. Huang, H. W. Luan, W. T. Dong, J. A. Fan, Q. L. Yang, J. A. Rogers, and Y. G. Huang, "Elasticity of Fractal Inspired Interconnects," Small, vol. 11, pp. 367-373, Jan 212015.

[105] W. Dong, C. Zhu, D. Ye, and Y. Huang, "Optimal design of self-similar serpentine interconnects embedded in stretchable electronics," Applied Physics A, vol. 123, p. 428, 2017.

[106] Y. Huang, W. Dong, T. Huang, Y. Wang, L. Xiao, Y. Su, and Z. Yin, "Self-similar design for stretchable wireless LC strain sensors," Sensors and Actuators A: Physical, vol. 224, pp. 36-42, 2015.

[107] Y. Su, Z. Liu, S. Kim, J. Wu, Y. Huang, and J. A. Rogers, "Mechanics of stretchable electronics with high fill factors," International Journal of Solids and Structures, vol. 49, pp. 3416-3421, 2012.

[108] S. Xu, Y. Zhang, L. Jia, K. E. Mathewson, K.-I. Jang, J. Kim, H. Fu, X. Huang, P. Chava, and R. Wang, "Soft microfluidic assemblies of sensors, circuits, and radios for the skin," Science, vol. 344, pp. 70-74, 2014.

[109] H. Son and C. Kim, "Multiimaging Sensor Data Fusion-Based Enhancement for 3D Workspace Representation for Remote Machine Operation," Journal of Construction Engineering \& Management, vol. 139, pp. 434-444, 2013.

[110] L. Xu, S. R. Gutbrod, Y. Ma, A. Petrossians, Y. Liu, R. C. Webb, J. A. Fan, Z. Yang, R. Xu, and J. J. Whalen, "Materials and fractal designs for 3D multifunctional integumentary membranes with capabilities in cardiac electrotherapy," Advanced Materials, vol. 27, pp. 1731-1737, 2015.

[111] C. H. Liu, G. F. Huang, C. H. Chiu, and T. Y. Pai, "Topology Synthesis and Optimal Design of an Adaptive Compliant Gripper to Maximize Output Displacement," Journal of Intelligent \& Robotic Systems, pp. 1-18, 2017.

[112] C. H. Liu, G. F. Huang, and T. L. Chen, "An evolutionary soft-add topology optimization method for synthesis of compliant mechanisms with maximum output displacement," Journal of Mechanisms \& Robotics, vol. 9, 2017.

[113] W. Dong, L. Xiao, C. Zhu, D. Ye, S. Wang, Y. Huang, and Z. Yin, "Theoretical and experimental study of 2D conformability of stretchable electronics laminated onto skin," SCIENCE CHINA Technological Sciences, vol. 60, p. 1415, 2017.

[114] H. Y. Cheng and S. D. Wang, "Mechanics of interfacial delamination in epidermal electronics systems," Journal of Applied Mechanics-Transactions of the Asme, vol. 81, Apr 2014.

[115] S. D. Wang, M. Li, J. Wu, D. H. Kim, N. S. Lu, Y. W. Su, Z. Kang, Y. G. Huang, and J. A. Rogers, "Mechanics of epidermal electronics," Journal of Applied Mechanics-Transactions of the Asme, vol. 79, May 2012.

[116] Y. Duan, Y. Huang, Z. Yin, N. Bu, and W. Dong, "Non-wrinkled, highly stretchable piezoelectric devices by electrohydrodynamic direct-writing," Nanoscale, vol. 6, pp. 3289-3295, 2014.

[117] J. Song, H. Jiang, Z. J. Liu, D. Y. Khang, Y. Huang, J. A. Rogers, C. Lu, and C. G. Koh, "Buckling of a stiff thin film on a compliant substrate in large deformation," International Journal of Solids and Structures, vol. 45, pp. 3107-3121, 2008. 
[118] D.-Y. Khang, J. A. Rogers, and H. H. Lee, "Mechanical Buckling: Mechanics, Metrology, and Stretchable Electronics," Advanced Functional Materials, vol. 19, pp. 1526-1536, 2009.

[119] S. Jung, J. H. Kim, J. Kim, S. Choi, J. Lee, I. Park, T. Hyeon, and D. H. Kim, "Reverse - micelle - induced porous pressure - sensitive rubber for wearable human-machine interfaces," Advanced Materials, vol. 26, pp. 4825-4830, 2014.

[120] H. K. Lee, J. Chung, S. I. Chang, and E. Yoon, "Normal and Shear Force Measurement Using a Flexible Polymer Tactile Sensor With Embedded Multiple Capacitors," Journal of Microelectromechanical Systems, vol. 17, pp. 934-942, 2008.

[121] C. Pang, G. Y. Lee, T. Kim, M. K. Sang, N. K. Hong, S. H. Ahn, and K. Y. Suh, "A flexible and highly sensitive straingauge sensor using reversible interlocking of nanofibres," Nature Materials, vol. 11, pp. 795-801, 2012.

[122] K. Takei, T. Takahashi, J. C. Ho, H. Ko, A. G. Gillies, P. W. Leu, R. S. Fearing, and A. Javey, "Nanowire active-matrix circuitry for low-voltage macroscale artificial skin," Nature materials, vol. 9, pp. 821-826, 2010.

[123] W. Dong, L. Xiao, W. Hu, C. Zhu, Y. Huang, and Z. Yin, "Wearable human-machine interface based on PVDF piezoelectric sensor," Transactions of the Institute of Measurement and Control, vol. 39, pp. 398-403, 2017.

[124] X. Wang, H. Zhang, R. Yu, L. Dong, D. Peng, A. Zhang, Y. Zhang, H. Liu, C. Pan, and Z. L. Wang, "Dynamic Pressure Mapping of Personalized Handwriting by a Flexible Sensor Matrix Based on the Mechanoluminescence Process," Advanced Materials, vol. 27, pp. 2324-31, 2015.

[125] W. Wu, X. Wen, and Z. L. Wang, "Taxel-addressable matrix of vertical-nanowire piezotronic transistors for active and adaptive tactile imaging," Science, vol. 340, pp. 952-7, 2013.

[126] X. Wang, H. Zhang, L. Dong, X. Han, W. Du, J. Zhai, C. Pan, and Z. L. Wang, "Self-Powered High-Resolution and Pressure-Sensitive Triboelectric Sensor Matrix for Real-Time Tactile Mapping," Advanced Materials, vol. 28, p. 2896, 2016.

[127] T. Bu, T. Xiao, Z. Yang, G. Liu, X. Fu, J. Nie, T. Guo, Y. Pang, J. Zhao, F. Xi, C. Zhang, and Z. L. Wang, "Stretchable Triboelectric-Photonic Smart Skin for Tactile and Gesture Sensing," Advanced Materials, vol. 0, p. 1800066.

[128] W. Hong, X. Zhao, and Z. Suo, "Large deformation and electrochemistry of polyelectrolyte gels," Journal of the Mechanics \& Physics of Solids, vol. 58, pp. 558-577, 2010.

[129] M. Shikida and K. Asano, "A Flexible Transparent Touch Panel Based on Ionic Liquid Channel," IEEE Sensors Journal, vol. 13, pp. 3490-3495, 2013.

[130] C. C. Kim, H. H. Lee, K. H. Oh, and J. Y. Sun, "Highly stretchable, transparent ionic touch panel," Science, vol. 353, p. $682,2016$.

[131] G. Gang, H. Wei, S. Jinjun, and D. Xiaochen, "Recent progress of flexible and wearable strain sensors for human-motion monitoring," Journal of Semiconductors, vol. 39, p. 011012, 2018.

[132] C. Lang, J. Fang, H. Shao, X. Ding, and T. Lin, "High-sensitivity acoustic sensors from nanofibre webs," Nature Communications, vol. 7, p. 11108, 2016.

[133] Y. Menguc, Y.-L. Park, H. Pei, D. Vogt, P. M. Aubin, E. Winchell, L. Fluke, L. Stirling, R. J. Wood, and C. J. Walsh, "Wearable soft sensing suit for human gait measurement," INTERNATIONAL JOURNAL OF ROBOTICS RESEARCH, vol. 33, pp. 1748-1764, Dec 2014.

[134] M. Trkov, J. Yi, T. Liu, and K. Li, "Shoe-Floor Interactions in Human Walking with Slips: Modeling and Experiments," Journal of biomechanical engineering, 2017 Oct 21 (Epub 2017 Oct 2017.

[135] B. J. Chen, E. H. Zheng, X. D. Fan, T. Liang, Q. N. Wang, K. L. Wei, and L. Wang, "Locomotion Mode Classification Using a Wearable Capacitive Sensing System," IEEE Transactions on Neural Systems and Rehabilitation Engineering, vol. 21, pp. 744-755, Sep 2013.

[136] E. Zheng, S. Manca, T. Yan, A. Parri, N. Vitiello, and Q. Wang, "Gait Phase Estimation Based on Noncontact Capacitive Sensing and Adaptive Oscillators," IEEE TRANSACTIONS ON BIOMEDICAL ENGINEERING, vol. 64, pp. 2419-2430, Oct 2017.

[137] Y. Qi, C. B. Soh, E. Gunawan, K. S. Low, and A. Maskooki, "A Novel Approach to Joint Flexion/Extension Angles Measurement Based on Wearable UWB Radios," IEEE Journal of Biomedical \& Health Informatics, vol. 18, p. 300, 
2014.

[138] S. Lim, D. Son, J. Kim, Y. B. Lee, J. K. Song, S. Choi, D. J. Lee, J. H. Kim, M. Lee, and T. Hyeon, "Transparent and Stretchable Interactive Human Machine Interface Based on Patterned Graphene Heterostructures," Advanced Functional Materials, vol. 25, pp. 375-383, 2015.

[139] J. B. Chossat, Y. Tao, V. Duchaine, and Y. L. Park, "Wearable soft artificial skin for hand motion detection with embedded microfluidic strain sensing," in IEEE International Conference on Robotics and Automation, 2015, pp. 2568-2573.

[140] T. Gulrez and A. Tognetti, "A Sensorized Garment Controlled Virtual Robotic Wheelchair," Journal of Intelligent \& Robotic Systems, vol. 74, pp. 847-868, 2014.

[141] J. Jeong, S. H. Bae, K. S. Min, J. M. Seo, H. Chung, and S. J. Kim, "A Miniaturized, Eye-Conformable, and Long-Term Reliable Retinal Prosthesis Using Monolithic Fabrication of Liquid Crystal Polymer (LCP)," IEEE transactions on biomedical engineering, vol. 62, pp. 982-9, 2015.

[142] N. Jaquier, M. Connan, C. Castellini, S. Calinon, N. Jaquier, M. Connan, C. Castellini, S. Calinon, N. Jaquier, and M. Connan, "Combining Electromyography and Tactile Myography to Improve Hand and Wrist Activity Detection in Prostheses," Technologies, vol. 5, p. 64, 2017.

[143] S. H. Jeong, K. Hjort, and Z. Wu, "Tape Transfer Atomization Patterning of Liquid Alloys for Microfluidic Stretchable Wireless Power Transfer," Sci Rep, vol. 5, p. 8419, 2014.

[144] Q. Hua, J. Sun, H. Liu, R. Bao, R. Yu, J. Zhai, C. Pan, and Z. L. Wang, "Skin-inspired highly stretchable and conformable matrix networks for multifunctional sensing," Nature Communications, vol. 9, p. 244, 2018/01/16 2018.

[145] J. Tamezduque, R. Cobianugalde, A. Kilicarslan, A. Venkatakrishnan, R. Soto, and J. L. Contrerasvidal, "Real-Time Strap Pressure Sensor System for Powered Exoskeletons," Sensors, vol. 15, pp. 4550-4563, 2015.

[146] D. Wentao, Z. Chen, H. Wei, X. Lin, and H. Yong'an, "Stretchable human-machine interface based on skin-conformal sEMG electrodes with self-similar geometry," Journal of Semiconductors, vol. 39, p. 014001, 2018.

[147] S. Han, M. K. Kim, B. Wang, D. S. Wie, S. Wang, and C. H. Lee, "Mechanically Reinforced Skin-Electronics with Networked Nanocomposite Elastomer," Advanced Materials, vol. 28, p. 10257, 2016.

[148] M. A. Yokus and J. S. Jur, "Fabric-Based Wearable Dry Electrodes for Body Surface Biopotential Recording," IEEE transactions on bio-medical engineering, vol. 63, p. 423, 2016.

[149] X. Zhang and Y. Jia, "A linear discriminant analysis framework based on random subspace for face recognition," Pattern Recognition, vol. 40, pp. 2585-2591, 2007.

[150] Y. Zhou, Y. Wang, R. Liu, L. Xiao, Q. Zhang, and Y. Huang, "Multichannel noninvasive human-machine interface via stretchable $\mu \mathrm{m}$ thick sEMG patches for robot manipulation," Journal of Micromechanics and Microengineering, vol. 28, p. $014005,2018$.

[151] L. Jin, H. Xian, Y. Jiang, Q. Niu, M. Xu, and D. Yang, "Research on Evaluation Model for Secondary Task Driving Safety Based on Driver Eye Movements," Advances in Mechanical Engineering, vol. 6, p. 624561, 2015.

[152] M.-H. Sigari, M.-R. Pourshahabi, M. Soryani, and M. Fathy, "A Review on Driver Face Monitoring Systems for Fatigue and Distraction Detection," International Journal of Advanced Science and Technology, vol. 64, pp. 73-100, 2014.

[153] S. Mishra, J. J. Norton, Y. Lee, D. S. Lee, N. Agee, Y. Chen, Y. Chun, and W. H. Yeo, "Soft, conformal bioelectronics for a wireless human-wheelchair interface," Biosensors \& Bioelectronics, vol. 91, pp. 796-803, 2017.

[154] X. Guo, W. Pei, Y. Wang, Y. Chen, H. Zhang, X. Wu, X. Yang, H. Chen, Y. Liu, and R. Liu, "A human-machine interface based on single channel EOG and patchable sensor," Biomedical Signal Processing and Control, vol. 30, pp. 98-105, 2016.

[155] R. M. Rothschild, "Neuroengineering Tools/Applications for Bidirectional Interfaces, Brain-Computer Interfaces, and Neuroprosthetic Implants - A Review of Recent Progress," vol. 3, p. 112, 2010.

[156] J. R. Wolpaw, N. Birbaumer, W. J. Heetderks, D. J. Mcfarland, P. H. Peckham, G. Schalk, E. Donchin, C. J. Robinson, and T. M. Vaughan, "Brain-computer interface technology: a review of the first international meeting," IEEE Transactions on Rehabilitation Engineering A Publication of the IEEE Engineering in Medicine \& Biology Society, vol. 8, pp. 164- 
173, 2000.

[157] A. Güneysu and H. L. Akin, "An SSVEP based BCI to control a humanoid robot by using portable EEG device," in Engineering in Medicine \& Biology Society, 2013, p. 6905.

[158] J. J. Norton, D. S. Lee, J. W. Lee, W. Lee, O. Kwon, P. Won, S. Y. Jung, H. Cheng, J. W. Jeong, and A. Akce, "Soft, curved electrode systems capable of integration on the auricle as a persistent brain-computer interface," Proceedings of the National Academy of Sciences of the United States of America, vol. 112, p. 3920, 2015.

[159] H. Kajimoto, M. Inami, N. Kawakami, and S. Tachi, "SmartTouch - Augmentation of Skin Sensation with Electrocutaneous Display," in Haptic Interfaces for Virtual Environment and Teleoperator Systems, 2003. Haptics 2003. Proceedings. Symposium on, 2003, pp. 40-46.

[160] M. Franceschi, L. Seminara, S. Dosen, M. Strbac, M. Valle, and D. Farina, "A system for electrotactile feedback using electronic skin and flexible matrix electrodes: Experimental evaluation," IEEE Transactions on Haptics, vol. PP, pp. 11, 2016.

[161] S. Kercel and P. Bach-Y-Rita, Human Nervous System, Noninvasive Coupling of Electronically Generated Data into: John Wiley \& Sons, Inc., 2006.

[162]K. A. Kaczmarek, "The tongue display unit (TDU) for electrotactile spatiotemporal pattern presentation," Scientia Iranica, vol. 18, pp. 1476-1485, 2011/12/01/ 2011.

[163] Vieira, L. Araújo, R. Dias, Sabino, and G. Schayer, "Biofeedback eletromiográfico (biofeedback/EMG) no pós-operatório de joelho," Fisioter Mov, vol. 20, 2007.

[164] T. Budzynski, "The Clinical Guide to Sound and Light," Zquavee Info, 2009.

[165] S. V. Da, A. P. Ribeiro, V. A. Dos Santos, A. E. Nardi, A. L. King, and M. R. Calomeni, "Stimulation by Light and Sound: Therapeutics Effects in Humans. Systematic Review," Clinical Practice \& Epidemiology in Mental Health, vol. 11, pp. 150-154, 2015.

[166] Y. Zhang, X. Geng, and H. Wang, "A System for Relieving Mental Fatigue Based on Light and Sound Stimulation," in Advanced Engineering Forum, 2012, pp. 228-233.

[167] M. Barnett-Cowan and L. R. Harris, "Temporal processing of active and passive head movement," Experimental Brain Research.experimentelle Hirnforschung.expérimentation Cérébrale, vol. 214, pp. 27-35, 2011.

[168] D. J. Young, M. A. Zurcher, M. Semaan, C. A. Megerian, and H. K. Wen, "MEMS Capacitive Accelerometer-Based Middle Ear Microphone," IEEE Transactions on Biomedical Engineering, vol. 59, pp. 3283-3292, 2012.

[169] T. I. Kim, J. G. Mccall, Y. H. Jung, X. Huang, E. R. Siuda, Y. Li, J. Song, Y. M. Song, H. A. Pao, and R. H. Kim, "Injectable, Cellular-Scale Optoelectronics with Applications for Wireless Optogenetics," Science, vol. 340, pp. 211-6, 2013.

[170] G. Shin, A. M. Gomez, R. Al-Hasani, Y. R. Jeong, J. Kim, Z. Xie, A. Banks, S. M. Lee, S. Y. Han, and C. J. Yoo, "Flexible Near-Field Wireless Optoelectronics as Subdermal Implants for Broad Applications in Optogenetics," Neuron, vol. 93, p. $509,2017$.

[171] S. I. Park, D. S. Brenner, G. Shin, C. D. Morgan, B. A. Copits, H. U. Chung, M. Y. Pullen, K. N. Noh, S. Davidson, and S. J. Oh, "Soft, stretchable, fully implantable miniaturized optoelectronic systems for wireless optogenetics," Nature Biotechnology, vol. 33, p. 1280, 2015.

[172] J. G. Mccall, T. Kim, G. Shin, X. Huang, Y. H. Jung, R. Alhasani, F. G. Omenetto, M. R. Bruchas, and J. A. Rogers, "Fabrication of flexible, multimodal light-emitting devices for wireless optogenetics," Nature Protocols, vol. 8, pp. 2413$28,2013$.

[173] B. C. Tee, A. Chortos, A. Berndt, A. K. Nguyen, A. Tom, A. Mcguire, Z. C. Lin, K. Tien, W. G. Bae, and H. Wang, "A skin-inspired organic digital mechanoreceptor," Science, vol. 350, p. 313, 2015.

[174] Y. Kato, S. Iba, T. Sekitani, and Y. Noguchi, "A flexible, lightweight Braille sheet display with plastic actuators driven by an organic field-effect transistor active matrix," in Electron Devices Meeting, 2005. IEDM Technical Digest. IEEE International, 2005 , pp. 4 pp.-100. 
[175] M. Takamiya, T. Sekitani, Y. Kato, H. Kawaguchi, T. Someya, and T. Sakurai, "Low Power and Flexible Braille Sheet Display with Organic FET's and Plastic Actuators," in IEEE International Conference on Integrated Circuit Design and Technology, 2006, pp. 1-4.

[176] Ankit, N. Tiwari, M. Rajput, N. A. Chien, and N. Mathews, "Highly Transparent and Integrable Surface Texture Change Device for Localized Tactile Feedback," Small, p. 1702312, 2017.

[177] S. Wei, T. Wang, and G. U. Guoying, "Design of a Soft Pneumatic Robotic Gripper Based on Fiber-reinforced Actuator," Journal of Mechanical Engineering, 2017.

[178] G. Y. Gu, U. Gupta, J. Zhu, L. M. Zhu, and X. Zhu, "Modeling of Viscoelastic Electromechanical Behavior in a Soft Dielectric Elastomer Actuator," IEEE Transactions on Robotics, vol. PP, pp. 1-8, 2017.

[179] C. Lee, M. Kim, Y. J. Kim, N. Hong, S. Ryu, H. J. Kim, and S. Kim, "Soft Robot Review," International Journal of Control Automation \& Systems, vol. 15, pp. 3-15, 2017.

[180] Y. Menguc, Y. L. Park, E. Martinez-Villalpando, P. Aubin, M. Zisook, L. Stirling, R. J. Wood, and C. J. Walsh, "Soft wearable motion sensing suit for lower limb biomechanics measurements," pp. 5309-5316, 2013.

[181] C. Cui, G. B. Bian, Z. G. Hou, J. Zhao, and H. Zhou, "A Multimodal Framework Based on Integration of Cortical and Muscular Activities for Decoding Human Intentions About Lower Limb Motions," IEEE Transactions on Biomedical Circuits \& Systems, vol. 11, pp. 889-899, 2017.

[182] J. G. San Juan, S. R. Gunderson, K. Kane-Ronning, and D. N. Suprak, "Scapular kinematic is altered after electromyography biofeedback training," Journal of Biomechanics, vol. 49, pp. 1881-1886, 2016.

[183] A. Sarasola-Sanz, N. Irastorza-Landa, E. López-Larraz, C. Bibián, F. Helmhold, D. Broetz, N. Birbaumer, and A. RamosMurguialday, "A hybrid brain-machine interface based on EEG and EMG activity for the motor rehabilitation of stroke patients," in International Conference on Rehabilitation Robotics, 2017, pp. 895-900.

[184] M. Schenk, "Family history and smoking predict pancreatic cancer risk," Clinical Rehabilitation, vol. 27, pp. 579-590, 2013.

[185] A. J. Westerveld, A. C. Schouten, P. H. Veltink, and d. K. H. Van, "Selectivity and resolution of surface electrical stimulation for grasp and release," IEEE Transactions on Neural Systems \& Rehabilitation Engineering A Publication of the IEEE Engineering in Medicine \& Biology Society, vol. 20, pp. 94-101, 2012.

[186] C. T. Freeman, "Electrode array-based electrical stimulation using ILC with restricted input subspace," Control Engineering Practice, vol. 23, pp. 32-43, 2014.

[187] N. M. Malešević, L. Z. P. Maneski, V. Ilić, N. Jorgovanović, G. Bijelić, T. Keller, and D. B. Popović, "A multi-pad electrode based functional electrical stimulation system for restoration of grasp," Journal of NeuroEngineering and Rehabilitation, vol. 9, p. 66, 2012.

[188] C. Ethier, E. R. Oby, M. J. Bauman, and L. E. Miller, "Restoration of grasp following paralysis through brain-controlled stimulation of muscles," Nature, vol. 485, pp. 368-371, 2012.

[189] P. L. Melo, M. T. Silva, J. M. Martins, and D. J. Newman, "Technical developments of functional electrical stimulation to correct drop foot: Sensing, actuation and control strategies," Clinical Biomechanics, vol. 30, pp. 101-13, 2015.

[190] G. Chai, X. Sui, S. Li, L. He, and N. Lan, "Characterization of evoked tactile sensation in forearm amputees with transcutaneous electrical nerve stimulation," Journal of Neural Engineering, vol. 12, p. 066002, 2015. 